\title{
From the synthesis of biobased cyclic carbonate to polyhydroxyurethanes: a promising route towards renewable NonIsocyanate Polyurethanes
}

\author{
Camille Carré, ${ }^{\mathrm{a}}$ Yvan Ecochard, ${ }^{\mathrm{b}}$ Sylvain Caillol ${ }^{\mathrm{b}}$, Luc Avérous $^{\mathrm{b} *}$

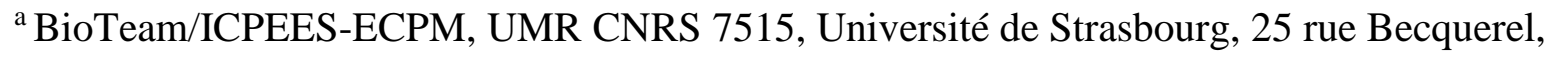 \\ 67087 Strasbourg Cedex 2, France \\ ${ }^{\mathrm{b}}$ ICGM, UMR 5253 - CNRS, Université de Montpellier, ENSCM, 240 Avenue Emile \\ Jeanbrau 34296 Montpellier, France \\ *Corresponding author: Prof. Luc Avérous, Phone: +33 3688527 84, Fax: +33 3688527 16, \\ Email: luc.averous@unistra.fr
}

\begin{abstract}
:
With a global production of around 18 million tons (6th among all polymers) and a wide range of applications such as rigid and soft foams, elastomers, coatings and adhesives, Polyurethanes (PUs) are one of the major polymer family. Nevertheless, they present important environmental and health issues. Recently, new and safer Polyurethanes, called NonIsocyanate Polyurethanes (NIPUs), have become a promising alternative to replace conventional PUs. Sustainable routes towards NIPUs will be discussed in this review from a green chemistry perspective. This paper will mainly focus on the reaction between biobased carbonates and amines which offers an interesting pathway leading to renewable Polyhydroxyurethanes (PHUs). The overview of the different routes for PHUs synthesis will draw attention to the green synthesis of cyclic carbonate (CC) compound and the aminolysis reaction. A state-of-the-art of different biobased building-blocks for PHUs synthesis will focus on $\mathrm{CC}$ compounds. Three classes of compounds will be defined according to the feedstock with (i) vegetable fats and oils, (ii) starch and sugars resources, and (iii) wood derivatives. Finally, biobased PHUs properties will be discussed.
\end{abstract}

Keywords: polyurethane; biobased polymer; cyclic carbonate; NIPU; non-isocyanate; polyhydroxyurethane 


\section{Introduction}

Crude oil and gas are traditionally used as feedstocks for the chemical industry. This strong dependence on fossil resources is being threatened by predictable shortages and price fluctuations in the upcoming years. In addition, global warming due to Carbon dioxide $\left(\mathrm{CO}_{2}\right)$ emissions and increasing environmental concerns lead chemical industries but also academic laboratories to develop new sustainable and safer chemical routes. ${ }^{[1]}$ Their current challenge is to substitute fossil resources by renewable ones to synthesize new promising molecules or building blocks for the elaboration of sustainable materials, especially new biobased polymers. ${ }^{[2]}$ Biobased polymers are defined as either partly (> 20\%) or fully based from biomass (e.g., from vegetal, animal and microorganisms). Biobased contents are mainly determined through the ${ }^{14} \mathrm{C}$ content, as fossil carbon resources do not contain anymore this isotope. ${ }^{[3]}$

Among the polymers, which can be biobased, and during the last decades, great attention has been particularly paid to the polyurethanes (PUs), which are one of the most common polymers used nowadays in industry. Based on their annual worldwide production of 18 million tons in 2016, PUs rank 6th among all polymers with a global market estimated at approximately 53 billion euros. ${ }^{[4]}$ Through their value chain, PUs involves four main players: (i) the chemists producing PUs raw materials, (ii) the producers of PUs from the raw materials, (iii) the compounders/assemblers who associate PUs into their final products, and finally (iv) the end-users. Due to the multiplicity of their structures and versatile properties, PUs can be used in various applications. They are mainly found as foams (rigid or flexible based on closed and open cells, respectively), but also as denser materials (elastomers, thermoplastics or thermosets). PUs can also be easily associated with other polymers to form blends, or with (nano)fillers/fibers to form (nano)composites. ${ }^{[5]}$ The main application of PUbased materials areas are in insulation, automotive, construction, household furniture and bedding, sports, coatings, packaging, fibers and textiles, electronics, footwear, biomedical and different appliances. ${ }^{[6]}$

Nowadays and in an agreement with more sustainable development, a great number of studies and developments have been realized in the field of biobased PUs. ${ }^{[7]}$ Since Otto Bayer in 1937, it is very well known that PU is commonly the product of the reaction of polyols and polyisocyanates. $^{[8]}$ Nowadays, both polyols and polyisocyanates can be biobased. Investigations have been particularly focused on biobased polyols (i) from fermentation of 
biomass (white biotechnologies) ${ }^{[9]}$ or (ii) directly extracted from biomass and then chemically modified, such as oleochemical resources. ${ }^{[7 \mathrm{c}, 7 \mathrm{~d}, 10]}$ Many industries (BASF, Cargill, Croda, Huntsman, Covestro, Avril-Novance) now produce their own grades of biobased polyols to bring different properties. ${ }^{[10 b]}$ On the other hand, different works on biobased isocyanates have also been published but to a much lesser extent. ${ }^{[7,, 11]}$ The main raw materials are vegetable derivatives (e.g. soybean or castor oil, and oleic acid). However, isocyanates from other renewable resources such as isosorbide ${ }^{[12]}$ have also been obtained. Some companies are currently emerging in the industrial synthesis of biobased isocyanates such as Cognis (BASFGermany), which produces 2-heptyl-3,4-bis(9-isocyanatononyl)-1-pentylcyclohexane from a dimer fatty acid, and Vencorex (Joint Venture between PTT Global Chemical - Thailand, and the Perstorp Group), which commercializes an aliphatic polyisocyanate named Tolonate ${ }^{\odot}$. All these recent developments show that fully biobased PUs could be produced in the near future. ${ }^{[13]}$

However, biobased polymers do not mean safe products. In this way, the second generation of environmentally PUs must also be non-toxic and non-hazardous, with a low impact on the environment. Then conventional PUs must be modified and new perspectives must be found. The emerging recent trend is the elaboration of NonIsocyanate PolyUrethanes (NIPUs) as isocyanates are harmful and suspected to be carcinogenic. ${ }^{[14]}$ Repetitive exposure can lead to skin irritation and incurable respiratory problems such as long-term asthma. Moreover, the synthesis of isocyanates requires the use of noxious substances such as phosgene. Since January 2009, a new European regulation was adopted, limiting the free isocyanate content in the medium. ${ }^{[14 b]}$ This regulation should be amplified and isocyanates could be banned or strictly restricted in the near future. Thus, three answers can be brought, such as (i) the reduction of isocyanate concentration in the media, (ii) the blocking of the remaining free isocyanate groups, or (iii) the replacement of isocyanates as chemical reagents. This latter approach, called the NIPU route, brings about new chemical pathways. Interest has grown for NIPUs and many papers have been published since the review of Guan et al. in 2011. ${ }^{[15]}$ Some general state of arts have been reported in the literature during the last decade. For instance, Mouloungui et al. or Cramail et al. have published a review in $2013^{[16]}$ and $2015^{[17]}$, respectively. On the same way, a short perspective paper has been recently published by Caillol et al. ${ }^{[4]}$ 
However, the aim of this review is focused on a biobased approach. This review is fully dedicated to a comprehensive overview of the synthesis and applications of biobased NIPUs, starting from the synthesis of biobased cyclic carbonate (CC). This paper is mainly focused on biobased NIPUs, which are synthesized via the aminolysis reaction, since this way is the most suitable route according to green chemistry principles. The aim of the "biobased PHUs" strategy is to identify promising routes for PHUs synthesis via the elaboration of efficient biobased CC. As a key compound, CC, as building blocks, is synthesized and selected in function of their availability, interest and limits. After a state of the art on NIPUs and PHUs synthesis, biobased building blocks for PHUs synthesis will be introduced. Then, PHUs materials and properties will be described. Finally, the future of these advanced materials will be reported and analyzed. All the references concerning CC and PHU syntheses are mainly related to the recent period, from the two last decades.

\section{Generalities about NIPU synthesis}

Even some other minor routes have been described in the literature, three main routes have been reported to synthesize NIPUs: (i) the AB-type azide condensation, (ii) the transurethane reaction, and (iii) the aminolysis. ${ }^{[15-16,18]}$ These different strategies, which are either polycondensation or polyaddition reactions, will be briefly described.

\subsection{NIPU synthesis via azide condensation and transurethanization}

\subsubsection{Main mechanisms}

The synthesis of NIPUs from azide compounds is an AB-type self-condensation between a hydroxyl $(\mathrm{OH})$ and an acyl azide group. The corresponding monomer should thus bring both of these functions. Acyl azides are most commonly prepared in excellent yield by acylation of sodium azide with acyl chlorides or carboxylic acids, previously activated with chloroformate

(Figure 1). ${ }^{[19]}$ Hydrazine derivatives obtained from ester can also be converted into acyl azide via an oxidation reaction (Figure 1). A Curtius rearrangement must be first achieved to convert the acyl azide into isocyanate.

The transurethanization process is another route for the synthesis of NIPUs. It is a two-step process with first the formation of diurethanes monomers (by conventional routes or aminolysis), and then the reaction with a diol in the presence of a suitable catalyst, to form the final polymer. Diurethane monomers used as reactants can be synthesized from isocyanates 
and $\mathrm{OH}$ groups or via the reaction between a carbonate and an aliphatic amine in the presence of a base. ${ }^{[20]}$ Yields from 22 to $100 \%$ (with suitable bases) can be obtained. ${ }^{[20 a, 20 b]}$. Nevertheless, the use of isocyanates to synthetize diurethanes does not match with the definition of NIPUs.

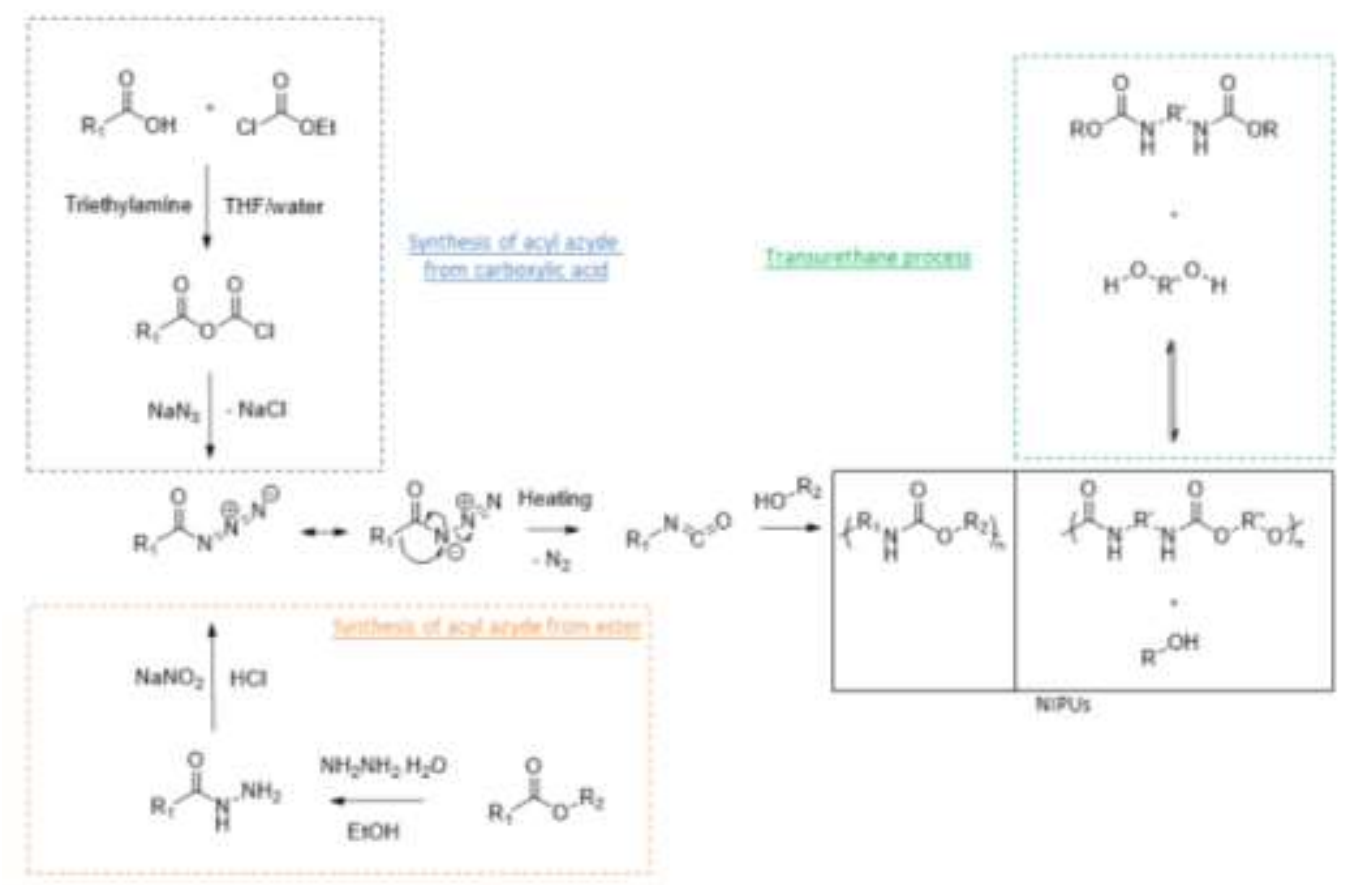

Figure 1. NIPU synthesis via azide condensation and transurethanization

\subsubsection{Advantages and drawbacks}

Biobased NIPUs from fatty acid derivatives bearing $\mathrm{OH}$ moieties, synthesized by carbon double bond epoxidation or thiol-ene chemistry, were recently developed and synthesized via the azide route. The obtained NIPUs show a thermoplastic behavior with a phase-separated morphology. ${ }^{[19 a, 21]}$ Numerous NIPU syntheses from the transurethanization process have been described in the literature and reviewed by Maisonneuve et al. ${ }^{[17]}$

Although isocyanate is not used as a starting reagent, these routes have nevertheless many disadvantages. The major inconvenient of the azide approach lies in the fact that the intermediate products are based on isocyanate groups, in opposition to the NIPU strategy. During transurethanization process synthesized alcohols must be extracted from the reaction medium to shift the equilibrium and increase the yield to $89 \% .{ }^{[20 \mathrm{~b}]}$ In addition, those methods are highly time and energy consuming. The Curtis arrangement requires a heating temperature 
between 80 to $100^{\circ} \mathrm{C}$, and reaction time from 10 to 24 hours. ${ }^{[19 a]}$ To obtain NIPUs by the transurethane reaction, diols must react with diurethanes, which require in the same way a relatively high temperature $\left(130-150^{\circ} \mathrm{C}\right)$.

Alcohol removal and the high reactions temperatures remain as major drawbacks for scale-up and industrialization. All these issues explain the very limited development and the literature about these routes to produce NIPUs and why a focus has been brought onto the PHUs synthesis route.

\subsection{PHUs synthesis via aminolysis reaction}

Aminolysis reaction is one of the main studied routes to synthesize NIPUs. This reaction involves the ring opening of a cyclic carbonate with an amine (Figure 2) which leads to a hydroxyurethane function and finally to polyhydroxyurethane (PHU) polymers. The following discussion will be mainly focused on the characteristics of the aminolysis and the current industrial applications. The discussion in this paper will be focused on CC synthesis as biobased intermediates from renewable resources. Diamines will not be described in this review.

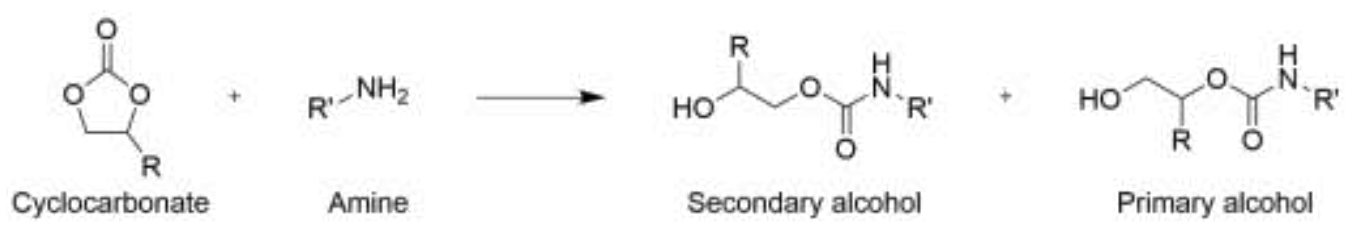

Figure 2. General Aminolysis reaction.

\subsubsection{The synthesis of cyclic carbonates}

The synthesis of $\mathrm{CC}$ has been initially studied more than sixty years ago. ${ }^{[22]}$ Nevertheless, industrial applications have only been developed over the last fifteen years. The most common 5-members CC (5CC) commercially available are presented in Figure 3. They are of great interest in the production of many products. ${ }^{[22]}$. However, 6-membered CC (6CC) and thiocarbonates exist and can also be involved as reactants for PHUs synthesis. 


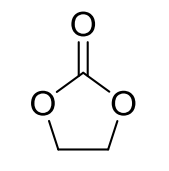

Ethylene carbonate

(EC)<smiles>CC1COC(=O)O1</smiles>

Propylene
carbonate

(PC)<smiles>O=C1OCC(CO)O1</smiles>

Glycerol carbonate

(GC)<smiles>C=CC1COC(=O)O1</smiles>

Vinyl Ethylene carbonate (VEC)

Figure 3. Most common 5CC.

\section{a) Main synthetic paths to cyclic carbonates}

Traditionally, the synthesis of CC was based on a chemical reaction between a diol and phosgene, or derivatives. ${ }^{[23]}$ The advantage of these reactions is the great reactivity of phosgene leading to high yields, even if they required additional purification steps. However, due to phosgene and the toxicity of its derivatives, ${ }^{[24]}$ attractive alternative approaches have been considered. The most common routes involving both diols and epoxides reactants are summarized in Figure $4 .{ }^{[25]}$ Only the routes presenting a green approach involving potential renewable resources will be discussed further.

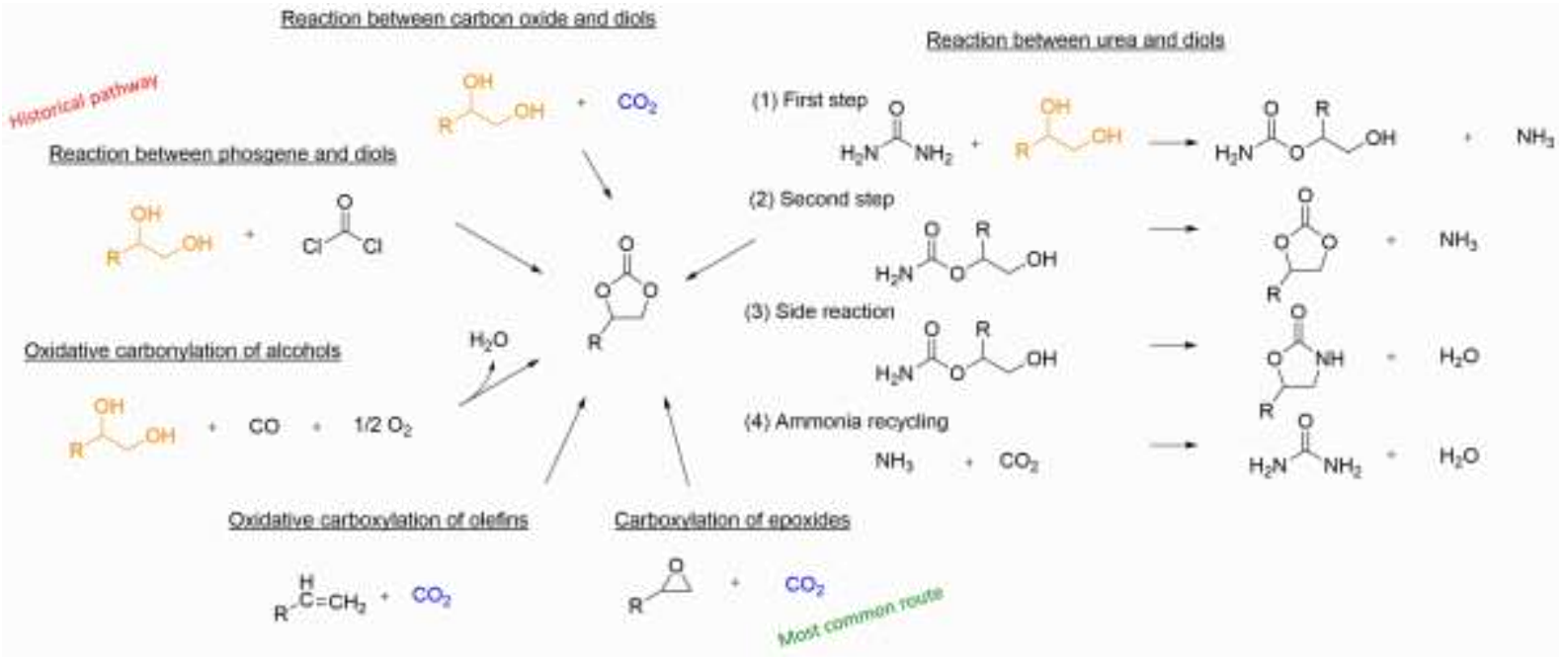

Figure 4. Chemical pathways to $\mathrm{CC}$ via potential renewable resources

Although less reactive than epoxides, diols can react to form $\mathrm{CC} . \mathrm{CO}_{2}$ is a sustainable resource and a naturally-occurring chemical compound. ${ }^{[26]}$ Therefore the production of CC from $\mathrm{CO}_{2}$ and various reactants, e.g. oxiranes and olefins, opens the field to fully biobased materials. ${ }^{[27]}$ 


\section{b) Oxidative carbonylation of alcohols}

Diols can be converted into $\mathrm{CC}$ by oxidative carbonylation with $\mathrm{CO}$ and oxygen as oxidant reagent (Figure 4). ${ }^{[28]}$ Heterogeneous catalysts have been studied for their efficiency of separation and recovery was compared to homogeneous catalyst systems. ${ }^{[29]}$

\section{c) The reaction between diols and urea}

$\mathrm{CC}$ are also synthesized from urea and diols, under reduced pressures and high temperatures. It is a two-step reaction with the formation of ammonia and an oxazolidone, in addition to the desired CC (Figure 4, reactions 1 to 3). ${ }^{[30]}$ Various diols were tested and substituted. Similarly, 6CC can be obtained. ${ }^{[31]}$ Furthermore the formed ammonia can be recycled by reaction with $\mathrm{CO}_{2}$ to produce water and urea, which can also be reused (Figure 4, reaction 4).

\section{d) The reaction between diols and carbonates}

The green synthesis of dimethyl carbonate (DMC) involves both methanol and carbon monoxide. Hence, glycerol carbonate (GC) can be obtained from glycerol and DMC by transesterification. A catalyst screening of this reaction has been reported by Ochoa-Gómez et $a l{ }^{[32]}$ High conversions and yields were reached with heterogeneous basic catalysts. Another green method, using molecular sieves but without other catalysts has also been developed leading to 5CC and 6CC. ${ }^{[33]}$ Furthermore, the catalyst-free improvement by thermal transesterification was investigated for batch and continuous-flow applications. ${ }^{[34]}$ Even if $\mathrm{DMC}$ is the most common chemical for this synthesis other carbonates like diphenyl carbonate can be used. ${ }^{[35]}$

\section{e) Synthesis of $\mathrm{CC}$ from epoxides and $\mathrm{CO}_{2}{ }^{[36]}$}

The current and industrial common pathway to synthesize $\mathrm{CC}$ is the bubbling of $\mathrm{CO}_{2}$ into oxiranes under high temperatures and pressures (Figure 5). $\mathrm{CO}_{2}$ is a sustainable resource coming from natural resources but also from industrial sources. $\mathrm{CO}_{2}$ can also be produced from the fermentation process. Furthermore, epichlorohydrin can be obtained from glycerol (via e.g. Epicerol ${ }^{\mathrm{TM}}$ process, Solvay) which makes this route a fully sustainable pathway. 


$$
\mathrm{O}_{\mathrm{R}}+\mathrm{N}\left(\mathrm{C}_{2} \mathrm{H}_{5}\right)^{4+} \mathrm{Br}^{-} \longrightarrow \mathrm{Br}^{-} \underbrace{\mathrm{N}\left(\mathrm{C}_{2} \mathrm{H}_{5}\right)^{4+}}_{\mathrm{R}}
$$

<smiles>[R]C(CBr)OC(=O)O[C@@H]1COC(=O)O1</smiles>

Figure 5. Mechanism synthesis of CC from epoxides. ${ }^{[22]}$

Carboxylation of epoxides was first reported in 1943 and continually published in the literature, more specifically on the catalysis systems. ${ }^{[37]}$ An alkylammonium halide catalyst is generally used to activate the epoxy ring. The solubility of $\mathrm{CO}_{2}$ in conventional solvents is low. In many cases, solvent-free reactions are achieved even if $\mathrm{CC}$ can be used as a solvent. Developments on other media were also performed. New alternatives are syntheses in supercritical $\mathrm{CO}_{2}\left(\mathrm{scCO}_{2}\right)$, in ionic liquids -as solvents and catalysts-, ${ }^{[38]}$ or both, combined. Reactions are achieved under mild temperatures, atmospheric pressures and enable energy and economic savings. Moreover, catalyst recovery is enhanced due to phase separation between $\mathrm{scCO}_{2}$ and a $\mathrm{CC}$, contrary to oxirane. Some new approaches such as microwave synthesis are also emerging. ${ }^{[39]}$

\section{f) Oxidative carboxylation of olefins}

Oxidative carboxylation of olefins is a direct conversion of olefins and $\mathrm{CO}_{2}$ into cyclic carbonates, in which preliminary formation and separation of epoxides are avoided (Figure 4). Since 1962, when this synthesis was first described ${ }^{[40]}$ numerous olefins have been studied. ${ }^{[41]}$ However, styrene remains the most reported one. Although syntheses based on different oxidants were also developed, Oxygen is the most commonly used. ${ }^{[41 \mathrm{a}, 42]}$ 
More recently, an efficient electrochemical method was developed; high yields of CC were obtained under mild conditions and without dangerous oxidant or expensive catalysts. ${ }^{[43]}$ Nevertheless, the literature specifies the utilization of short olefins such as styrene and does not lead to multifunctional monomers for materials synthesis. The $\mathrm{CO}_{2}$ carbonation of epoxides obtained via $\mathrm{H}_{2} \mathrm{O}_{2}$ oxidation of olefins is largely preferred, in particular to obtain carbonated triglyceride oils.

\section{g) The reaction between $\mathrm{CO}_{2}$ and diols}

Diols can react with $\mathrm{CO}_{2}$ in supercritical conditions to form $\mathrm{CC}$ and water (Figure 4). Various catalysts such as metal oxides or organic bases were reported to promote the reaction. ${ }^{[44]}$ All catalytic systems present high selectivity, however yields still remain low and are, in addition to necessary water removal, the main drawback of this reaction.

\section{h) Synthesis of six-, seven- or eight-membered CC}

In addition to standard $5 \mathrm{CC}, 6 \mathrm{CC}$ or higher rings can also be synthesized. $6 \mathrm{CC}$ can be obtained by reaction with oxetanes (Figure 6).

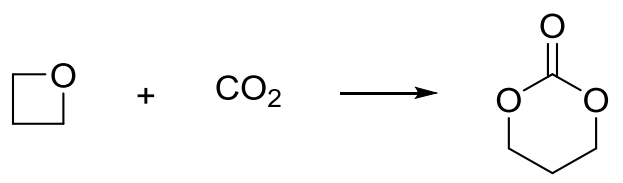

Figure 6. Synthesis of a 6CC from an oxetane.

Oxetanes are four-membered cyclic ethers, and due to larger ring sizes, are more stable than oxiranes. Consequently, the addition of $\mathrm{CO}_{2}$ is less efficient. The first synthesis of $6 \mathrm{CC}$ from oxetane and $\mathrm{CO}_{2}$ was reported in $1985 .{ }^{[45]}$

Besides cycloaddition from oxetanes, several routes for the synthesis of higher CC rings were reported. Instead of 1,2-diols involved in the synthesis of 5CC by oxidative carbonylation, 1,3-diols can also be used to obtain 6CC. The main source of carbonyls are however phosgene derivatives. ${ }^{[46]} 7 \mathrm{CC}$ were also synthesized from 1,4-diols and triphosgene by Tomita et al. ${ }^{[47]}$ They demonstrated that $6 \mathrm{CC}$ or $7 \mathrm{CC}$ are more reactive than conventional $5 \mathrm{CC}$. N-substituted 8CC, synthesized with N-methyldiethanolamine and triphosgene, showed even higher reactivity towards amines. ${ }^{[48]}$ However, due to the more difficult preparation of $6 \mathrm{CC}$, larger cycles are less employed in the synthesis of PHUs. Their main application is ring-opening 
polymerization (ROP). A biobased 7CC, obtained from tartric acid was synthesized from 1,4diols and triphosgene with pyridine in THF at room temperature. ${ }^{[49]}$ Carbonate exchange reaction between alcohol and carbonate sources (phosgene, alkylene carbonate or dialkyl carbonate) in the presence of a suitable catalyst is another pathway for $\mathrm{CC}$ synthesis. ${ }^{[50]}$ Recently, enzymatic transesterification reaction with lipase, for a greener chemistry was reported. ${ }^{[51]}$ Reaction between diols and dimethyl carbonate can also lead to 6CC via the same pathway than $5 \mathrm{CC} .{ }^{[33]}$ Despite the possibility to find biobased diols, phosgene derivatives are major precursors for $6 \mathrm{CC}$ and $7 \mathrm{CC}$ synthesis. The use of such dangerous precursors for our health and the environment is incompatible with sustainable chemistry principles. Thus, $5 \mathrm{CC}$ are largely preferred for PHUs synthesis.

\section{Coupling reactions}

Another method to obtain $\mathrm{CC}$ monomers is to directly graft GC onto monomers. A first coupling method for the synthesis of $\mathrm{CC}$ is the esterification of an acid or an acyl halide with GC, a CC having an $\mathrm{OH}$ group. ${ }^{[52]}$ This method is based on using glycerol as it is a byproduct of oleochemistry and biodiesel production. This route is interesting for the synthesis of biobased PHUs. GC and numerous acids are $100 \%$ biobased and the final compounds are easily obtained without the use of strong catalysts or harsh conditions. However, this chemical pathway is more sensitive to hydrolysis reactions, due to the presence of ester bonds in the chain. New chemistry derived from thiol derivatives was developed to overcome this problem. For example, Annunziata et al. have developed GC telechelic precursors via the reaction of GC tosylate with alcohols. ${ }^{[53]}$ The reaction between vinyl $\mathrm{CC}$ and thiols is encountered in many recent studies. ${ }^{[25,47,54]}$ The commercially available vinyl ethylene carbonate is the main precursor of this chemistry.

\subsubsection{Aminolysis reaction}

PHUs are synthetized through a polyaddition between amines and cyclic carbonates. To understand the condition of PHU synthesis, the aminolysis of the cyclic carbonates with an amine, and the different parameters affecting this reaction are firstly report. The main parameters affecting the polymerization reaction are detailed. 


\section{a) Main mechanisms}

The reactivity between a CC and an amine group has been largely studied by Garipov et al. ${ }^{[55]}$ They propose a three-step reaction mechanism (Figure 7).

(1)

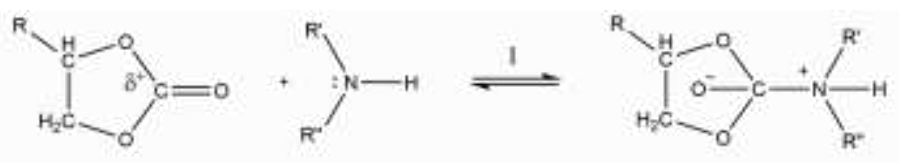

(2)

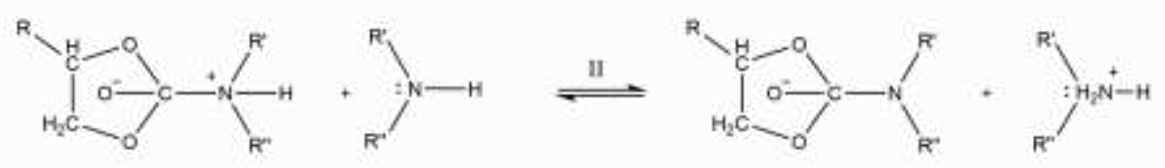

(3)

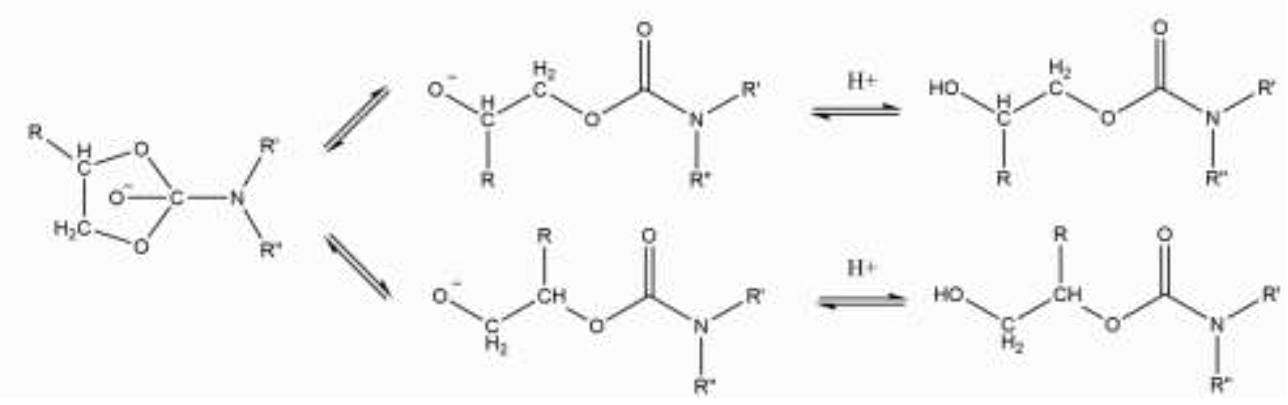

Figure 7. Mechanism of aminolysis reaction. ${ }^{[55]}$

The first step of the reaction is the nucleophilic attack of the amine on the carbonyl group of the $\mathrm{CC}$, resulting in the formation of a tetrahedral intermediate. Then, the tetrahedral intermediate is deprotonated in a second step by another amine. The last step, promoted by the strong electron withdrawing effect of the nitrogen atom, is the rupture of the carbon-oxygen bond of the $\mathrm{CC}$, which leads to ring-opening and formation of an alkoxyl ion. Then, the latter rapidly recombines with a proton to form the products of the reaction. Thus, $\mathrm{CC}$ reacts with amine to form an urethane bond, or more precisely an hydroxyurethane, since $\mathrm{OH}$ groups are also synthesized. According to the ring-opening mechanism, primary and secondary $\mathrm{OH}$ groups, are formed (Figure 7).

\section{b) Parameters affecting the aminolysis}

\section{The structure of the amine}

The amine reactivity is governed by the chemical structure and the molar mass. ${ }^{[56]}$ Indeed, low molar mass amines, as well as aliphatic and non-substituted amines, show a higher 
reactivity when compared to aromatic and substituted ones. Furthermore, $\mathrm{OH}$ group on the amine increases its nucleophilic character and thus higher reaction rate are obtained. Secondary amines are less reactive than primary ones, and no reaction occurs with tertiary amines. ${ }^{[57]}$

\section{The structure of the CC}

It appears from several studies, ${ }^{[58]}$ that the nature of the substituents on the CC influences its reactivity towards diamines. It was shown that the reaction is accelerated with electronwithdrawing groups. Indeed, they decrease the electron density of the carbon of the carbonyl bond. In addition, they allow the formation of predominant secondary $\mathrm{OH}$ groups. It corresponds to an increase of the acidity of the negatively charged oxygen by inductive effect. In addition to electronic effect, steric hindrance and hydrogen bonding between carbonate and amine are impacting carbonate reactivity as well. The substituent impacts the kinetic according the following ranking: ethyl-ester > acetate > trimethylhexanoate > benzoate, ethylether > phenyl-ether > butane. ${ }^{[4,59]}$ The reactivity scale of different $\mathrm{CC}$ are given Figure 8. Carbonates of different structures reacted with an amine (EDR-148). The kinetic studies were followed by ${ }^{1} \mathrm{H}$ NMR. Cyclic carbonates was compared according to their reactivities, based on kinetic measurements.

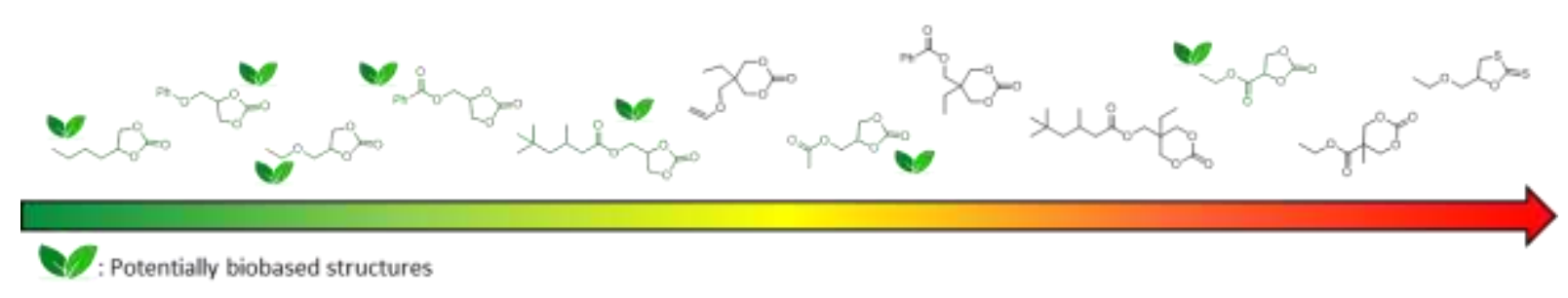

Figure 8. Reactivity scale of various CC structures ${ }^{[59]}$

$\mathrm{CC}$ size and structure are also key parameters. $8 \mathrm{CC}$ and thiocarbonates are more reactive but they all involve harmful reactants in their synthesis (phosgene and derivatives or carbon disulfide) ${ }^{[4]}$ Although $5 \mathrm{CC}$ are the least reactive, they are the easiest to synthesize and the most used. Due to the nature of biobased compounds and green routes towards $\mathrm{CC}, 5 \mathrm{CC}$ seems to be the best choice. 


\section{Effect of the solvent of the reaction}

Garipov et al. have shown that the aminolysis reaction is more favored in presence of protic solvents. ${ }^{[55]}$ However, in the literature, reactions are mainly carried out in aprotic solvents. In these cases, the reaction rate increases with increasing polarity of the medium. Furthermore, the limiting step of the reaction depends on the nature of the solvent. When the reaction is carried out in protic solvents, the limiting step is the deprotonation. On the contrary, in aprotic solvents, the reaction rate-limiting step is the nucleophilic attack of the amine on the CC. More recently, a complete kinetic study of the aminolysis reaction has shown that protic solvents have a positive impact on reactivity, conversion of cyclic carbonates, and the molar mass of the synthesized PHUs via a quasi-quantitative conversion. ${ }^{[4]}$ Nowadays, the major trend is to perform PHUs synthesis in bulk for greener applications.

\section{Effect of the molar ratio between CC and amines}

It was determined that systems synthesized at equimolar ratio present PHUs with the highest properties. At non-stoichiometric ratio, secondary reactions and hydrogen bonding can yield oligomers with low molar masses. ${ }^{[60]}$ However, maintaining an equimolar ratio during PHUs synthesis is very challenging due to the numerous possible side reactions, ${ }^{[17]}$ which impacts the monomer conversion and the molar masses.

\section{Influence of the reaction temperature and polymerization time}

PHUs synthesis at room temperature has been reported, but increasing the temperature largely enhances the reactivity. Viscosity increases during polymerization as well as the amount of hydrogen bonds. Thus, a rise of temperature can overcome this issue leading to higher reaction rates. Temperature of PHUs synthesis should not exceed $120^{\circ} \mathrm{C}$ to avoid side reactions such as amidification. ${ }^{[4,17]}$ Depending on the polymerization temperature, reactions can last from few hours to several days.

\section{c) Catalysis systems}

The kinetics of PHUs synthesis is often described as very slow. ${ }^{[61]}$ This is particularly due to the relatively stable nature of $\mathrm{CC}$. To catalyze the aminolysis reaction, either electrophilicity of the carbonyl group on the CC (acid catalysts), or the nucleophile character of the amine (basic catalysts) can be increased. 


\section{Basic catalysts}

In the literature, the main basic catalysts for aminolysis are piperazine and triethylamine. Triazabicyclodecene (TBD) seems also to be promising. ${ }^{[62]}$ generally used for ROP and transesterifications, TBD allows to synthesize PHUs with molar masses around 30,000 g.mol ${ }^{-}$ ${ }^{1}$ within 24 hours. A high value compared to previously reported ones. ${ }^{[63]}$

\section{Other catalyst systems (salts and organo-based catalysts)}

Ochiai et al. ${ }^{[64]}$ have examined the influence of different salts (acids and bases) on the final molar mass of the PHUs, on the conversion rate of bisCC and on the secondary/primary $\mathrm{OH}$ ratio in the chain. They show that whatever the catalyst, the conversion of bisCC is increased. However, basic salt catalysts have shown to yield higher conversion for salts with monovalent cations. For acid catalysts, impact varies depending on the salt. ${ }^{[64]}$ Although tin salts are predominantly used as a catalyst in the literature, it would be preferred to use lithium salts which are equally reactive and less harmful with regards to health and environment.

More recently, inorganic salts and organo-catalysts have been compared for coupling propylene carbonate and cyclohexylamine. ${ }^{[65]}$ Organo-catalysts such as TBD and thiourea present higher catalytic activities compared to inorganic Lewis acids.

\section{d) Side reactions}

Numerous side reactions have been described in the literature leading to the creation of several byproducts and non-reactive chain ends. The changes on reactant stoichiometry could explain the conversion limitations and the difficulty to reach high molar mass, limiting PHU development. Schemes of those reactions are presented Figure 9, based on model structures of cyclic carbonate and amine. 


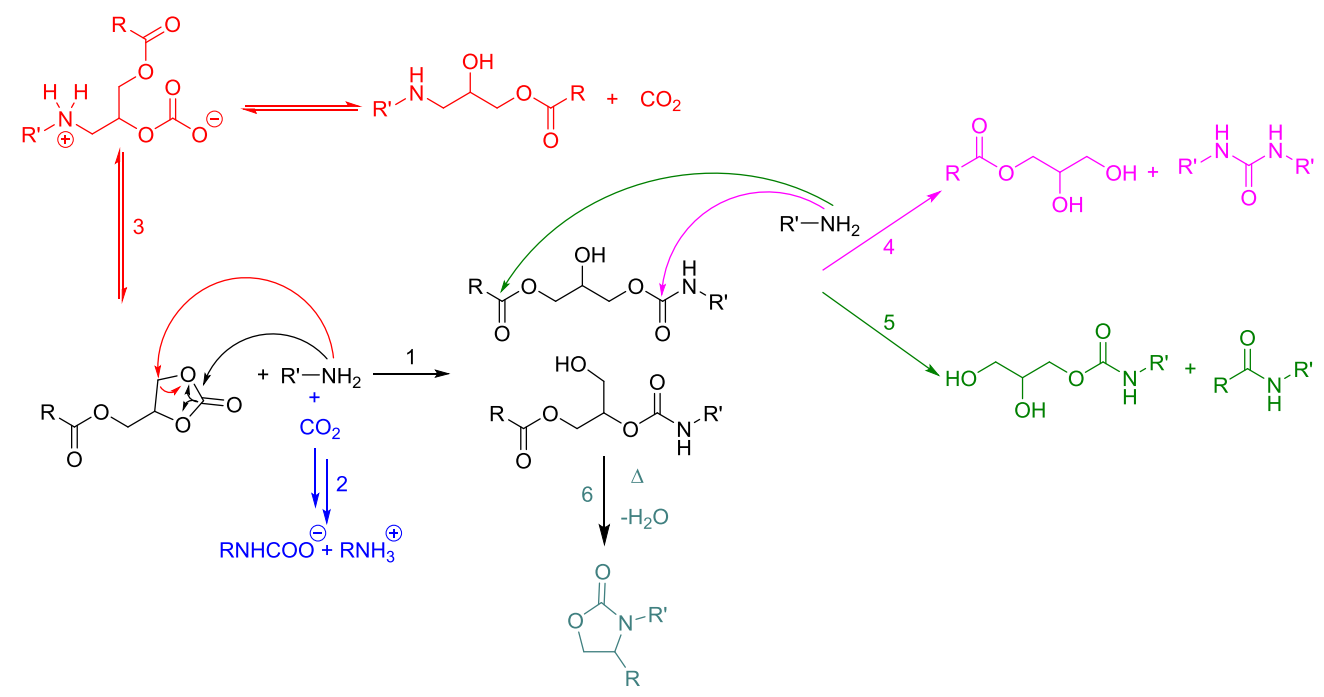

Figure 9. Possible reactions between 5CC and amine: (1) conventional aminolysis, (2) carbonation of amine, (3) CO2-in-situ formation, (4) urea formation by trans-urethanization, (5) amidification reaction and (6) oxazolidinone formation by dehydration ${ }^{[4]}$

\section{General side reactions}

At high temperatures (above $100^{\circ} \mathrm{C}$ ), amines can react with the formed hydroxyurethane moieties to yield unreactive diols and ureas. ${ }^{[66]}$ The reaction pathway can be described in two steps. Recently, it has been shown that such trans-urethane reactions leading to urea formation are also observed under catalyst-free conditions and at $80^{\circ} \mathrm{C}$, which is the most commonly used temperature in PHUs synthesis. ${ }^{[67]}$ Clements et al. first described the formation of cyclic oxazolidinones from the reaction between an amine and a CC. ${ }^{[22]}$ Recently, the formation of oxazolidinones as a side reaction of the synthesis of PHUs via aminolysis has been reported. ${ }^{[67]}$ The attack of amines on $\alpha$-carbon of the carbonate group can lead to in-situ formation of $\mathrm{CO}_{2}$. Presence of $\mathrm{CO}_{2}$ from in-situ formation or from the atmosphere may cause the formation of carbonate anions and ammonium salts. The amine can also attack the urethane group formed after aminolysis by trans-urethanization leading to urea formation. ${ }^{[67]}$ Furthermore, the surprising presence of glycerol as by-product of an aminolysis reaction was recently highlighted, ${ }^{[68]}$ after the decomposition of GC by a hydrolysis process. The glycerol yield largely depends on both the amine reactivity and the water content in the media.

All these side-reactions reduce the amine content and cause divergence from the equimolar stoichiometry in addition to ending the polymer chain growth and are responsible for the low 
molar masses. Nevertheless, these reactions generally occur above $100^{\circ} \mathrm{C}$ or in presence of catalysts.

\section{Synthesis of amide groups}

Ester groups are currently found in biobased compounds and particularly in the synthesis routes of biobased CC monomers. Thus, amidification is a major concern for biobased PHUs synthesis. If the $\mathrm{CC}$ is synthesized by the reaction between carboxylic acid or its derivatives and GC, the formed ester is then able to react with an amine to form an amide. However, this reaction is not always detected. For instance, Tamami et al. do not show any amide groups by

${ }^{13} \mathrm{C}$-NMR and FTIR spectroscopy, at room temperature. ${ }^{[69]}$ However, amide formation was confirmed at conventional temperatures for an aminolysis reaction (i.e., from 70 to $100^{\circ} \mathrm{C}$ ), as shown by the amide peak presence at $1636-1643 \mathrm{~cm}^{-1}$. This secondary reaction is particularly highlighted in the case of an amine excess with regards to cyclic carbonate. ${ }^{[60 \mathrm{~b}]}$ Furthermore, esters functions are particularly present in vegetable oils and are susceptible to undergo amidification. In the case of carbonated soybean oil, Javni et al. studied the competition between the urethane and the amide formation as well as the influence of the amine structure. ${ }^{[60 \mathrm{~b}]}$ Boyer et al. have highlighted this sub-reaction in the case of a fatty diester compound with internal or terminal carbonates. ${ }^{[70]}$

\section{Formation of hydrogen bonding}

Recently, Blain et al. showed by DSC and NMR that low molar masses are not only due to the occurrence of side reactions like urea formation, but mainly due to hydrogen bonding. ${ }^{[17}$, ${ }^{71]}$ DSC reveals the presence of a second enthalpy of reaction in addition to the enthalpy of reaction of the CC/amine system. This explains why full conversion of monomers cannot be reached and thus the low molar mass of PHUs. NMR and additional DSC analyses evidence that hydrogen bonding plays a key role in PHUs polymerization and the hydrogen bonding network is mainly responsible for the second enthalpy of reaction. Compared with conventional PUs, PHUs with their $\mathrm{OH}$ functions can easily develop hydrogen bonding. In order to reduce inter- and intra-molecular hydrogen bonding plasticizers can be used. Chain mobility will be then increased and monomer conversion will be improved to yield a higher degree of polymerization. 


\section{e) Main advantages and drawbacks of the aminolysis reaction}

The synthesis of NIPUs seems to show promising alternatives to conventional PU syntheses. However, all the main paths have advantages and disadvantages. ${ }^{[19 a, 20 b, 72]}$ Although a long reaction time is required for the aminolysis reaction (several days), this synthetic route is the most studied for the elaboration of NIPUs. Indeed, aminolysis is less restrictive and more industrially transferable compared to e.g., pathways based on transurethane reaction or ABtype azide condensation.

Several monomers and catalysts were tested. Then, conversion rates were increased, especially at the beginning of the reaction, but do not reach more than $80 \%$ with very low degrees of polymerization and low molar masses. To conclude, the main obstacles include the use of (noxious) solvents in order to control the synthesis and important reaction times (several days), currently impeding industrial development. Synthesis temperatures are quite modulable (especially for the aminolysis, from room temperature to $100^{\circ} \mathrm{C}$ ).

Nowadays, more and more PHUs materials are developed towards industrial applications, mainly where low molar masses are not a disadvantage. Similar to conventional PUs, PHUs exhibit a large range of architectures for numerous applications. In addition to thermosets and thermoplastics, PHUs are now available as foams, hydrogels or latex. ${ }^{[4]}$

\section{Biobased PHUs}

\subsection{Building blocks from renewable resources}

In a context of reducing crude oil consumption and carbon footprints with the environment preservation, biorefineries have emerged to replace fossil resources by renewable feedstocks. Different types of biomass can be used (e.g., animal, vegetal, algae, mushroom) to provide a large number of chemicals and polymers. ${ }^{[73]}$ The most exploited biomass source is vegetal, mainly from forestry and crops with different types of waste or residues. Feedstocks can be classified into different groups: lignocellulose, vegetable fats and oils, starch and sugars (Figure 10).

Herein, the potential and efficiency of biobased building blocks for NIPUs via aminolysis reaction are reviewed. 


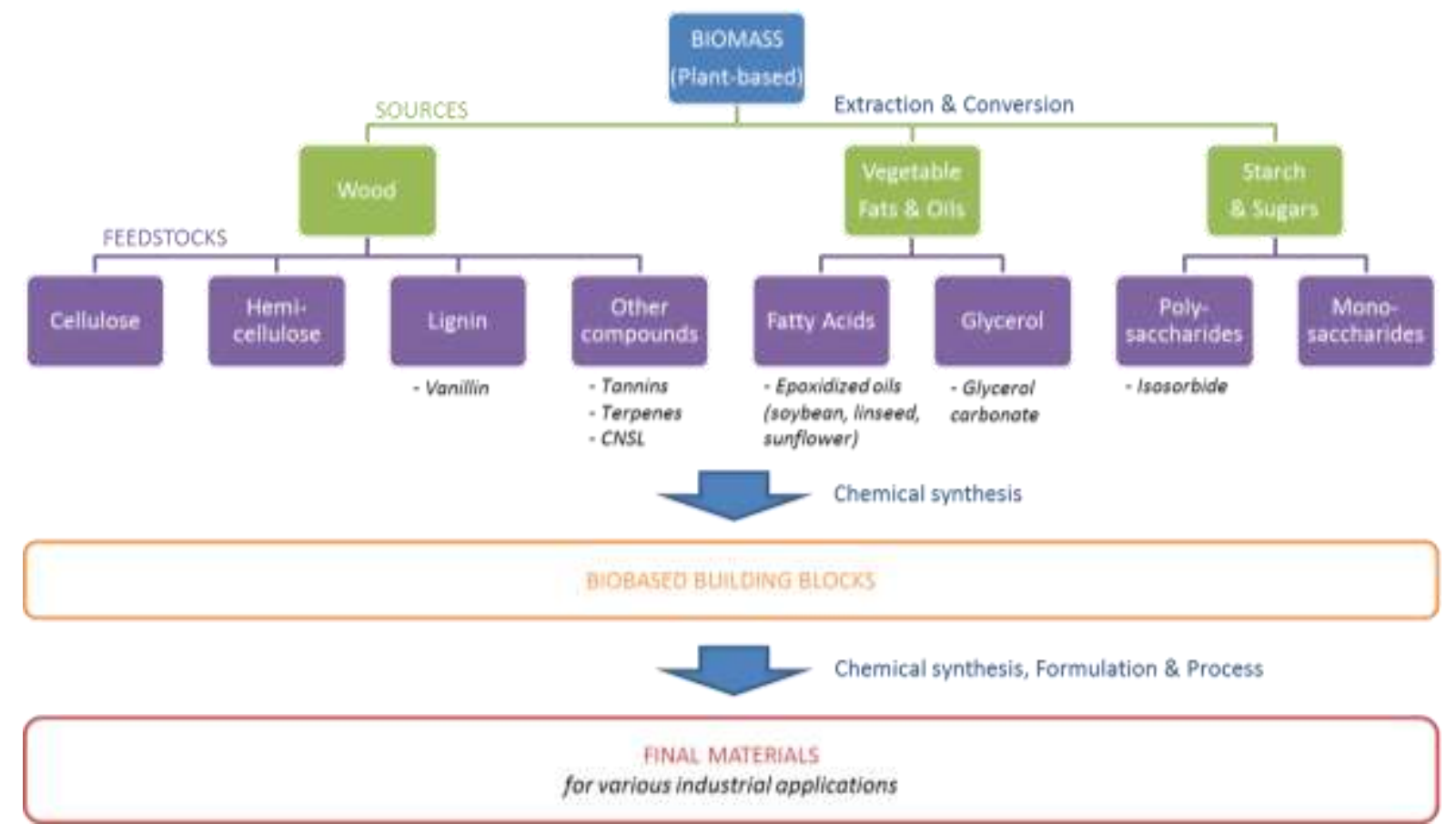

Figure 10. From plant-based biomasses to building blocks and end-materials

Various routes to $\mathrm{CC}$ have been developed concurrently with the investigation and the developments on NIPUs. The main strategy involves the carbonation of epoxide. More and more new CC are biobased. Besides, diamines for PHU can be biobased. They are more and more often commercialized. Recently, Pelckmans et al. have reported a complete review on biobased diamines. ${ }^{[74]}$

In this section, we will focus on the various routes towards biobased building blocks for PHU synthesis and particularly on biobased $\mathrm{CC}$ as the key compound of this system. $\mathrm{CC}$ and some diamines will be classified according to the origin of the corresponding renewable resources: (i) vegetable fats and oils, (ii) starch and sugars, and (iii) lignocellulosic feedstock.

\subsubsection{Vegetable oils}

Vegetable oils are mainly based on triglycerides. The composition of fatty acids in triglycerides varies and depends on the botanical source and the environment. ${ }^{[75]}$ In addition to their carboxylic acid end groups, fatty acids can present one to several double bonds, and can also bring $\mathrm{OH}$ and epoxy units, such as castor and vernonia oil, respectively. ${ }^{[76]} \mathrm{A}$ rich oleo-chemistry ${ }^{[10 b]}$ associated with a large and global availability make this resource very attractive for the synthesis of innovative biobased monomers and building blocks. 


\section{a) Synthesis of $\mathrm{CC}$ and diamines from vegetable oils}

\section{Cyclic carbonates}

All CC from triglyceride derivatives are synthesized from carbonation of epoxidized vegetable oils (EVO) by $\mathrm{CO}_{2}$ (Figure 11). EVO are molecules of interest due to their reactive oxirane sites but also their low prices and are readily available. Epoxidation is generally applied on the double bonds with an oxidizing agent, typically a peracid (e.g., peracetic acid). Microwaves oven may be used to accelerate the epoxidation, thus transforming fatty acid derivatives into epoxides in a few minutes at a high yield. ${ }^{[77]}$ In order to work in mild conditions and improve selectivity, reactions can also be performed by chemo-enzymatic process with immobilized lipases. ${ }^{[78]}$

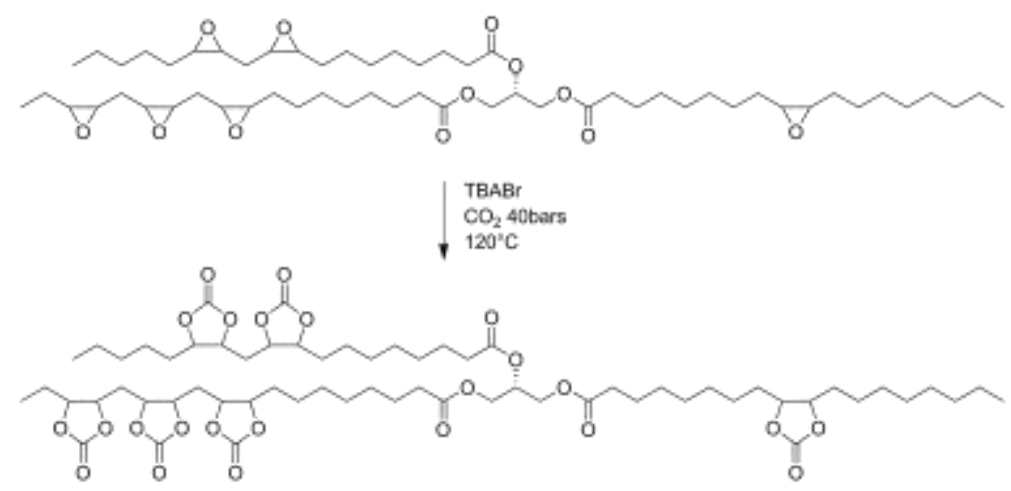

Figure 11. Carbonation of epoxidized vegetable oils

Epoxidized soybean oil (ESBO) and epoxidized linseed oil (ELSO) are commercially available and commonly used in a large range of applications. Various new epoxidized vegetable oils from e.g. cottonseed, ${ }^{[79]}$ rapeseed, ${ }^{[80]}$ Lesquerella and Limnanthes (Meadowfoam) ${ }^{[81]}$ are also emerging. Carbonation of EVO is always performed with $\mathrm{CO}_{2}$, at gaseous or supercritical state. EVO carbonation process has been recently summarized. ${ }^{[82]}$ Even if oxiranes are highly reactive groups, their position inside the triglyceride chains can induce steric hindrance and considerably reduce the conversion of epoxy compounds into carbonates. In order to promote the reaction, TBAB is widely used as a catalyst for the synthesis of EVO but high temperature and pressure, as well as several hours, are still required to obtain a high conversion. ${ }^{[60,69,79,83]}$ Some progress were realized using a binary catalyst system $\mathrm{SnCl}_{4} \cdot 5 \mathrm{H}_{2} \mathrm{O} / \mathrm{TBAB}$, which combines a Lewis acid (for epoxide activation) and a Lewis base $\left(\mathrm{CO}_{2}\right.$ activation). ${ }^{[84]}$ Other catalysts such as $\mathrm{KI}$ activated by 18 -crown- $6,{ }^{[85]}$ 
$\mathrm{SiO}_{2}-\mathrm{I},{ }^{[86]}$ or TBAB with addition of water ${ }^{[87]}$ were also tested but no satisfactory result were obtained. Carbonation reactions are typically carried out between 60 and $180^{\circ} \mathrm{C}$ during 10 to 170 hours. $\mathrm{ScCO}_{2}$ improves reagent solubility and decreases viscosity, ${ }^{[88]}$ then reaction time is more than twice as short compared with reaction at atmospheric pressure. Optimization of reaction conditions is thus required in the future to consider a large-scale production.

Functionality of the carbonated vegetable oils strongly affects the stiffness and the $\mathrm{T}_{\mathrm{g}}$ of PHUs materials synthesized from carbonated soybean oil (CSBO) when cured with aliphatic amines leading to soft and flexible materials with $\mathrm{T}_{\mathrm{g}}<20^{\circ} \mathrm{C}$. Even with short triamine such as tris(2-aminoethyl)amine (TDA) CSBO-based materials remain soft $\left(\mathrm{T}_{\mathrm{g}}=6^{\circ} \mathrm{C}, \mathrm{E}=3.7\right.$ $\mathrm{MPa}) .{ }^{[89]}$ The use of carbonated linseed oil (CLSO) as unique monomers or mixed with CSBO increases the stiffness of materials with $\mathrm{T}_{\mathrm{g}}$ up to $60^{\circ} \mathrm{C}$ and Young modulus above 1000 $\mathrm{MPa} .{ }^{[86]}$ Webster et al. developed biobased and highly functional CC from epoxidized sucrose soyate (combination of sucrose and vegetable oils fatty acids) under supercritical conditions. ${ }^{[90]}$ They optimized the synthesis conditions for coating applications by varying the catalysts, solvents and curing process. The solvent resistance of the coatings has been improved by the choice of a dual catalyst system Lewis acid/Lewis base and the use of protic solvents.

In the literature, most of the $\mathrm{CC}$ come from triglycerides and present functionalities higher than two and are thus not adapted to the synthesis of linear biobased PHUs. To straighten out this issue, Cramail et al. prepared CC from well-defined oleic acid methyl ester. ${ }^{\left[70,{ }^{91]}\right.}$ They also demonstrate that terminal epoxides are much more reactive than internal ones, frequently encountered in the case of triglycerides carbonation. In the same way, the reactivity of internal carbonates is also reduced. Moreover, because of this low reactivity and the presence of ester functions, the amidification side-reaction is enhanced. ${ }^{[60 \mathrm{~b}]}$

\section{Diamines}

Only few papers report the synthesis of diamine from vegetable oils. The functionalization of grapeseed oil with amine groups involving cysteamine hydrochloride and the thiol-ene reaction. ${ }^{[92]}$ Dimer fatty amines result from the dimerization (Diels-Alder mechanism) of fatty acids followed by an amination reaction. Fully biobased dimer diamines are commercially available building blocks with low viscosity, high flexibility and low $\mathrm{T}_{\mathrm{g}}$. These properties 
make them suitable for the synthesis of various biobased polymers. ${ }^{[93]}$ Such diamines were recently reported in PHU synthesis, ${ }^{[91]}[52 \mathrm{~d}]$.

\section{b) Synthesis of cyclic and bis(cyclic carbonates) from glycerol and derivatives}

Glycerol, which was identified in 2004 as a top added value chemical from biomass by the US Department of Energy, is one of the most prevalent biobased wastes (e.g., from the biodiesel industry). ${ }^{[94]}$ It is a platform building block for various derivatives including CC. ${ }^{\text {[94-95] }}$ Depending on which substituents are present on the carbonate ring, mainly $\mathrm{OH}$ or vinyl functional groups, esterification or thiol-ene coupling reactions can be envisioned to obtain bifunctional CC.

\section{GC and derivatives}

GC is a cheap, biodegradable and non-toxic renewable compound, which has gained interest over the past 20 years for its versatile reactivity and applications. It is currently considered as a promising biobased building block. ${ }^{[96]}$

As reviewed recently (Figure 12), ${ }^{[96]}$ GC can result from various, rather simple synthetic routes. All the reactions were similar to conventional syntheses of $\mathrm{CC}$.

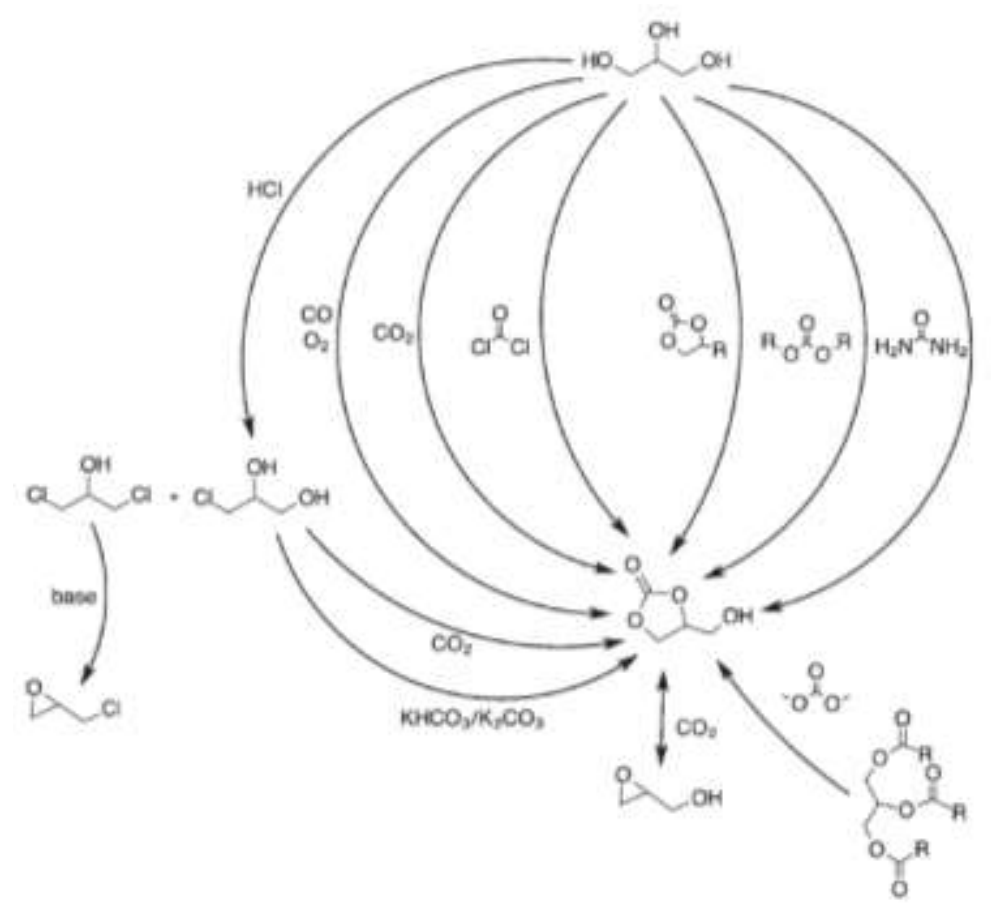

Figure 12. Main routes to GC. ${ }^{[96]}$ 
Reactions involving $\mathrm{CO}_{2}$ with glycerol or epoxides are environmentally interesting because resulting from waste products from different industries. However, the uses of organic solvents and catalysts, as well as harsh temperature and pressure conditions, are strong issues for the environment. Recently, Ochoa-Gomez et al. ${ }^{[97]}$ have shown that the reaction between glycerol and $\mathrm{CO}_{2}$ is the most promising process. However, it cannot at this time be considered for a large-scale production of $\mathrm{GC}$ due to low $\mathrm{CO}_{2}$ reactivity, which consequently yields low conversion rates. Novel routes such as enzymatic transesterification reaction of glycerol with dimethyl carbonate ${ }^{[98]}$ or enzymatic coproduction of biodiesel and GC by transesterification of soybean oil and dimethylcarbonate in bulk are also emerging. ${ }^{\text {[99] }}$

In addition to its relatively straightforward synthesis, GC has a wide reactivity due to the presence on the same molecule of two units, a primary $\mathrm{OH}$ and a 2-oxo-1,3-dioxolane group (ODO). GC thus possesses nucleophilic and electrophilic sites. The ODO group can react with many nucleophilic compounds such as $\mathrm{OH}$, amines, carboxylic acids, ketones, or isocyanates groups. The $\mathrm{OH}$ group of GC shows a similar reactivity to ODO group. Selectivity is thus an important parameter to avoid undesired side reactions.

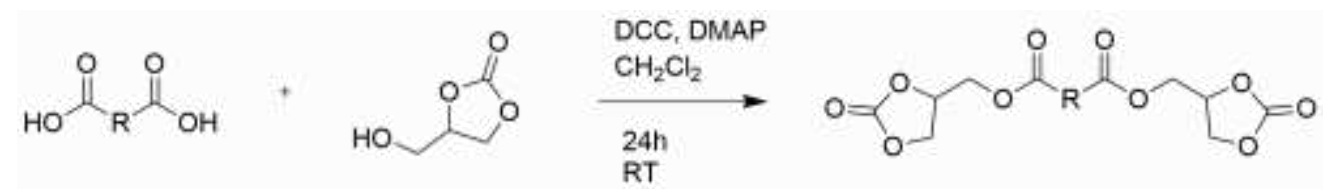

Figure 13. Coupling reaction between GC and carboxylic acid

CC can be grafted through coupling reactions between GC and a diacid (Figure 13). Guillaume et al. have polymerized trimethylene carbonate with propane-diol. Then, the resulting polycarbonate was modified with succinic anhydride to obtain polyesters with acid chain ends. The addition of GC on diacid leads to polyester oligomers bearing $\mathrm{CC} .{ }^{[52 \mathrm{c}]} \mathrm{By}$ a green chemical pathway i.e. without solvent and catalysts, sebacic-bisCC has been synthesized via the reaction between GC and sebacoyl chloride. Amines with functionality around 2.2 have been used as cross-linkers to obtain promising elastomers with high elongation $(>600 \%) .{ }^{[52 \mathrm{~d}]}$

On the other hand, diglycerol, an oligomer of glycerol, gives access to bifunctional compound via transesterification process with dimethylcarbonate. A yield of $79 \%$ has been reached with potassium carbonate. However, a selective separation through crystallization is necessary and 
could impede possible scale-up. ${ }^{[100]}$ Higher yields (88\%) have recently been obtained with a strong catalyst, $\mathrm{Li}-\mathrm{La}_{2} \mathrm{O}_{3} \cdot{ }^{[101]}$ The use of such monomer with various diamines to obtain fully biobased PHUs has been reported for coatings with promising wood adhesive properties. ${ }^{[102]}$

\section{Vinyl functional alkylene carbonates}

Vinyl functional alkylene carbonates can also be prepared from glycerol. GCs functionalized with vinyl reactive groups have been formed under a Williamson ether synthesis followed by a palladium reduction (Figure 14). ${ }^{[103]}$ The cyclic-acyclic carbonate intermediate has been isolated with $57 \%$ yield and the final vinyl carbonate with $82 \%$ yield. This protocol can be used to obtain a bisCC via thiol-ene chemistry. ${ }^{[25]}$

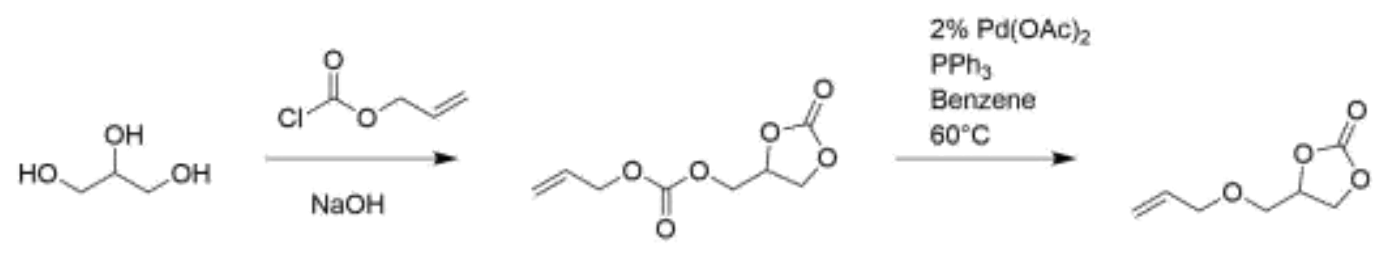

Figure 14. Vinyl ethylene GC synthesis.

GC and acryloyl chloride, in dichloromethane using triethylamine as a catalyst, lead to GC acrylate, another CC resulting from GC. ${ }^{[104]}$

\subsubsection{Starch and sugars resources}

\section{a) The case of isosorbide (ISO)}

With two $\mathrm{OH}$ functional groups per molecule, isosorbide (ISO or 1,4:3,6-dianhydrosorbitol) is a promising renewable platform chemical for the synthesis of biobased polymers. ${ }^{[105]}$ Furthermore, its two cycloaliphatic rings increase the rigidity of the architectures. ISO is commercially available from starch. The ISO production from biomass occurs in a multistep protocol. ${ }^{[106]}$ First, glucose from various polysaccharides is hydrogenated to obtain sorbitol (SOR), which is then converted into ISO via a dehydration step. Alternative productions have been developed from non-edible lignocellulosic biomass. 
Carbonate synthesis

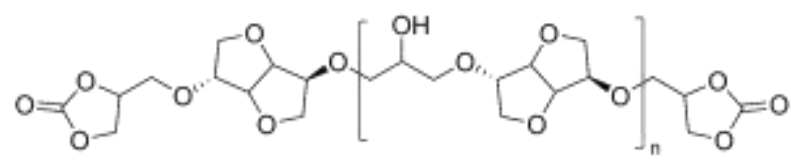

Figure 15. Structure of an oligomer of ISO carbonate

Synthesis of epoxys and amines from ISO have been largely studied and developed for epoxy resins ${ }^{[107]}$ and other polymers. ${ }^{[106]}$ However, from our knowledge, a sole study reports the use of ISO in PHU synthesis. ${ }^{[108]}$ The authors prepared ISO CC in three steps. They synthesized the diglycidyl ether of ISO through allylation and epoxidation with meta-chloroperoxybenzoic acid. ISO diglycidyl ether oligomers are also synthesized via a second route with an excess of epichlorohydrin in the presence of sodium hydroxide. Finally, a $\mathrm{CO}_{2}$ carbonation reaction occurs to form the desired ISO di(cyclic carbonate) (Figure 15), which then reacts with various diamines to obtain ISO-based PHUs. Materials with $\mathrm{T}_{\mathrm{g}}$ from -8 to $59^{\circ} \mathrm{C}$ are synthesized with PPG-diamine (JEFF400), diethylentriamine, decanediamine and isophorondiamine (IPDA), leading to softer or stiffer material, respectively.

\section{Amine synthesis}

Even if ISO-derived diamine was not used in PHU synthesis, such compounds already exist. The first diamine synthesized from ISO was described in 1946. ${ }^{[109]}$ The diamine resulted from a tosylation of $\mathrm{OH}$ groups from ISO followed by a nucleophilic substitution with methyl alcoholic ammonia, performed in an autoclave. Other methods starting from tosylation reaction were also reported. Thiem et al. successively performed azide formation and hydrogenation using palladium as a catalyst. ${ }^{[110]}$ Van Es et al. realized a nucleophilic substitution on tosylated ISO with benzylamine followed by a catalytic hydrogenolysis. ${ }^{[111]}$ Other ISO bifunctional amine derivatives were also developed. Fluorobenzene compounds were used to achieve first a nucleophilic aromatic substitution, and then a reduction with Raney Nickel or a catalytic hydrogenation in order to obtain a diamine. ${ }^{[112]}$ More recently, the synthesis of ISO-based diaminopropyl was proposed in two steps, involving first the reaction of ISO with acrylonitrile, and then hydrogenation with Raney Nickel. ${ }^{[113]}$ 
To conclude, ISO is a potential chemical a source of amines or epoxides, for PHUs. Most notably epoxide carbonation techniques that lead to $\mathrm{CC}$ are the most well-known.

\section{b) Other sugar derivatives}

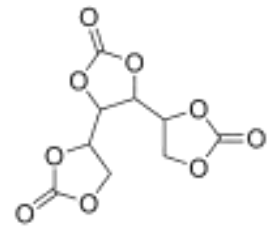

Sorbitol tricarbonate STC

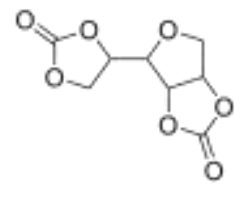

Sorbitol bis(cyclic carbonate)

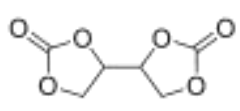

Erytritol bis-carbonate

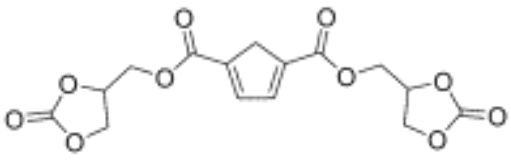

2,5-furandicarboxylic acid-based bis(cyclic carbonate)

Figure 16. Different structure of $\mathrm{CC}$ from sugar derivatives

\section{Sorbitol (SOR)}

SOR, an intermediate building block has also been studied for the synthesis of carbonates (Figure 16). SOR-based tricarbonate has been recently obtained through two different ways. $^{[14]}$ The first way was performed via a direct carbonation of the three vicinal diols groups with diphenyl carbonate. The second way involves four steps with (1) a dimerization with acrolein, (2) a first carbonation with diethyl carbonate, (3) an epoxidation of the olefin groups via m-CPBA, (4) and then a final carbonation with $\mathrm{CO}_{2}$. This tricarbonate was finally formulated with two different biobased amines for coating applications. In 2017 they used commercial SOR-based glycidyl ether to obtain a new green polyfunctional carbonated monomer via $\mathrm{CO}_{2}$ carbonation. This new compound is easily handled thanks a low viscosity, a good miscibility with amines and a liquid state at room temperature. Mixing these two SORbased $\mathrm{CC}$ with IPDA, $\mathrm{T}_{\mathrm{g}}$ and Young's modulus of around $180^{\circ} \mathrm{C}$ and $4000 \mathrm{MPa}$ were obtained respectively, leading to one of the stiffest PHUs materials ever reported. ${ }^{[15]}$ Recently, a new route towards bisCC from SOR has been fully investigated with improved conversion and limited side reactions with respect to green chemistry principles (solvent-free, low temperature and catalyzed reactions). Optimum results were obtained with TBD as catalyst and a continuous feed of DMC into the reactional medium. Fully biobased PHUs materials were finally synthesized with short and biobased diamines. ${ }^{[116]}$ 


\section{Erythritol}

Synthesis of erythritol from glucose was performed by yeast fermentation. Through a direct carbonation of diphenyl carbonate or via $\mathrm{CO}_{2}$ carbonation of the 2,2'-bioxirane, obtained by epoxidation of bioethanol-derived butadiene, erythritol dicarbonate can be obtained with $90 \%$ yield (Figure 16) without solvents or byproducts. Thanks to a solid state and a high reactivity biobased PHUs have been formulated by melt-processing, mixing erythritol dicarbonate with different biobased diamines. ${ }^{[35]}$ Reactive processing requires much lower reaction times and gives access to high molar masses compared to conventional polymerizations in solution. Reactive extrusion or injection molding are very interesting in terms of efficient industrial applications.

\section{2,5-Furandicarboxylic acid}

Furandicarboxylic acid (FDCA) derived from cellulosic biomass is a promising renewable compound for PHUs synthesis. Zhang et al. have described a novel method to obtain 2,5furan-bisCC via a three-step procedure (Figure 16). ${ }^{[117]}$ The FDCA is reacted with allyl bromide via the dehydrobromination reaction followed by an epoxidation with $\mathrm{m}$ chloroperoxybenzoic acid. Finally, the diglycidyl ester of FDCA is carbonated with $\mathrm{CO}_{2}$ leading to 2,5-furan-bisCC.

\subsubsection{Bioresources from wood}

\section{a) Lignin}

Lignin is a polymeric aromatic material. Along with cellulose, it is one of the major components of the cell walls of wood and plants. Lignin is considered as a polyphenol with different $\mathrm{OH}$ groups. Nowadays, it is one of the main aromatic renewable resources for the elaboration of aromatic chemicals and polymers. Recently, lignin was used for the first time in the elaboration of PHUs. ${ }^{[18]}$ First, urethane monomers were prepared via aminolysis reaction between carbonated soybean oil and an aminosilane-coupling agent. Then, they were polymerized with lignin to form PHUs. PHUs with high biomass contents (up to 85\%) were obtained. Moreover, it was observed that increasing the lignin content in the material enhances the tensile strength (up to $1.4 \mathrm{MPa}$ ). Direct modifications of lignin have been 
recently performed leading to epoxidized lignin derivative by reaction of epichlorohydrin. Then, carbonated lignin was obtained by $\mathrm{CO}_{2}$ carbonation. ${ }^{[119]}$ Cross-linked materials have been thereafter reported with the use of carbonated lignin with diaminododecane and PEG-CC as a chain extender. ${ }^{[120]}$ By tuning the ratio between PEG-C5 and lignin-based CC, polymers with tailored properties are obtained, ranging from soft elastomer to rigid thermosets resin. The curing process at $80^{\circ} \mathrm{C}$ during $20 \mathrm{~h}$ with THF has led to materials with $\mathrm{T}_{\mathrm{g}}$ from -35 to $15^{\circ} \mathrm{C}$. A novel approach has also been described by Kühnel et al. via a coupling reaction by two steps with GC. ${ }^{[121]}$ First, an oxyalkylation of lignin leads to diols functions, which are in a second step carbonated through transesterification with DMC, for a conversion up to $96 \%$. This environmentally friendly protocol is effective under mild conditions $\left(75^{\circ} \mathrm{C}, 6 \mathrm{~h}\right)$ in the presence of catalytic amounts of $\mathrm{K}_{2} \mathrm{CO}_{3}$.

\section{b) Tannins}

Tannins are the second most abundant source of natural aromatic compounds, after lignins. Tannins are phenolic molecules mainly found in trees and plants. They can also be used in the elaboration of NIPUs. Recently, Pizzi et al. reported the synthesis of NIPUs from various tannins, ${ }^{[122]}$ via aminolysis reaction, after a preliminary carbonation of tannin with dimethylcarbonate (Figure 17). Soluble parts of the mixture have been analyzed through MALDI-TOF analysis and SEC to identify the products of the reaction. They have been used as coatings on wood surface to improve hydrophobicity.
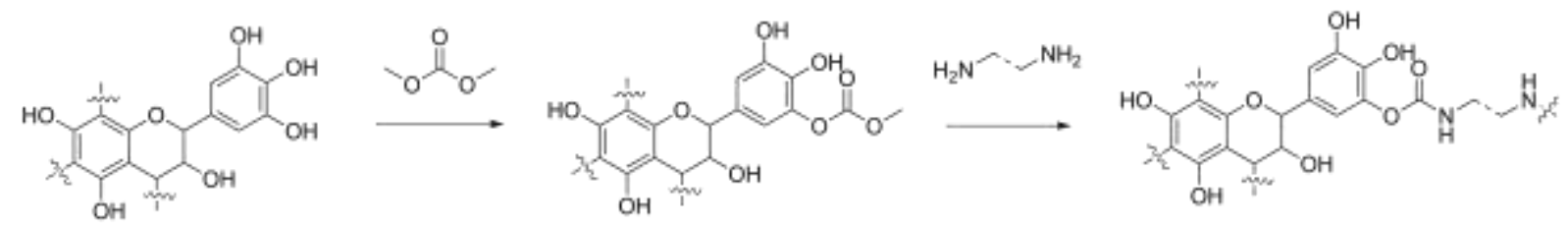

Figure 17. Route toward NIPUs from tannins

Gallic acid has also been used for CC synthesis and mixed with epoxidized Polyhedral Oligomeric Silsesquioxanes (POSS) and amines yielding PHU/POSSs materials. ${ }^{[123]}$

\section{c) Vanillin from lignin}

Vanillin is a natural compound extracted from Vanilla orchids seed pods. Due to its limited availability, different synthetic paths have been developed. ${ }^{[124]}$ Biobased vanillin can be 
obtained from sulfonated lignin from black liquor from paper process. However, due to harsh conditions (e.g. severe oxidative process and intensive purification procedure), vanillin is mainly obtained from guaiacol, based on fossil resources. ${ }^{[124 a]}$ Biobased vanillin and its derivatives (Figure 18) can be considered as aromatic platform chemicals, as they enable the synthesis of various monomers, including diamines and CC. ${ }^{[124 a]}$

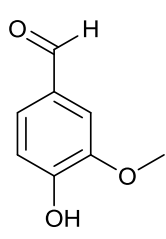

Vanillin

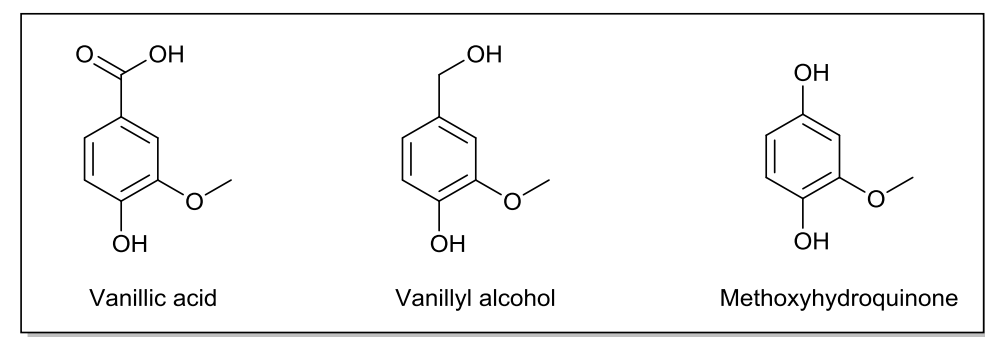

Figure 18. Vanillin and its derivatives.

Caillol et al. is the first team to work on the elaboration of CC from biobased vanillin. They reported the synthesis of vanillin-based diamines, via thiol-ene addition of cysteamine hydrochloride. ${ }^{[124 a]}$ The synthesis is carried out in two steps. First, epichlorohydrin reacts with $\mathrm{OH}$ moieties to form an epoxide, and then addition of $\mathrm{CO}_{2}$ induces the epoxide ring-opening to obtain the desired CC. To obtain vanillin-derived epoxide, greener methods were developed to replace Bisphenol-A and epichlorohydrin. ${ }^{[125]}$ This two-step method involves alkaline allylation of $\mathrm{OH}$ groups followed by epoxidation of the resulting carbon double bond, either via m-chloroperoxybenzoic acid or via hydrogen peroxide with lipase as catalyst. The main advantage is to avoid oligomerization which always occurs with the use of epichlorohydrin. Oligomerization is nevertheless a good way to decrease viscosity of monomers. Indeed, vanillin diglycidyl ether is solid while vanillin diglycidyl ether oligomers are liquids and are thus easy to process.

\section{d) Other wood derivatives}

\section{Cardanol from Cashew Nut Shell Liquid (CNSL)}

CNSL is the oil contained in the shell of cashew nut (Figure 19). As wastes from the cashew nut industry, CNSL and its derivatives are not in competition with food. It is a promising biobased viscous liquid, largely available, with low toxicity and cost and a production of one million tons per year. ${ }^{[126]}$ 


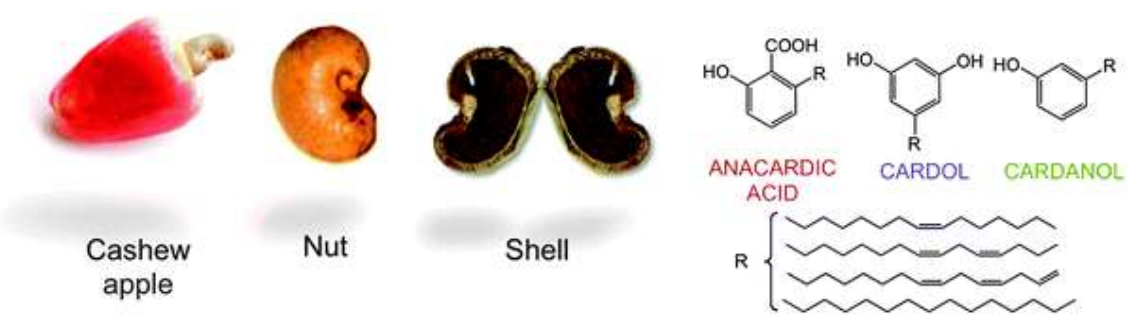

Figure 19. From cashew apple to cardanol. ${ }^{[127]}$

Various methods including roasting and hot vegetable oil process are used to extract CNSL from the nut, with respective variations of the chemical composition. ${ }^{[128]}$ Main constituents are anacardic acid, cardol and cardanol; aromatic compounds containing phenolic moieties and unsaturated carbon side chains (Figure 19). During the thermal treatment and the following refining distillation step, decarboxylation of anacardic acid occurs to obtain cardanol. The three reactive sites of cardanol $(\mathrm{OH}$ group, aromatic ring and unsaturated aliphatic chain) make it a promising building block to substitute fossil-based phenol derivatives, and as a versatile raw material for monomers and polymer synthesis. ${ }^{[129]}$ For instance, cardanol is a biobased alternative to Bisphenol-A, ${ }^{[107]}$ and to polyols for polyurethanes. $^{[130]}$

As far as we know, CC from CNSL was only reported by Kathalewar et al. for the synthesis of PHU coatings. ${ }^{[131]}$ Commercial NC514 epoxidized cardanol (from Cardolite) reacts with $\mathrm{CO}_{2}$ under pressure using TBAB as catalyst (Figure 20). Coatings of PHUs materials have been obtained with amines, IPDA and hexamethylendiamine (HMDA) and compared to the corresponding epoxy-based materials (NC-514).

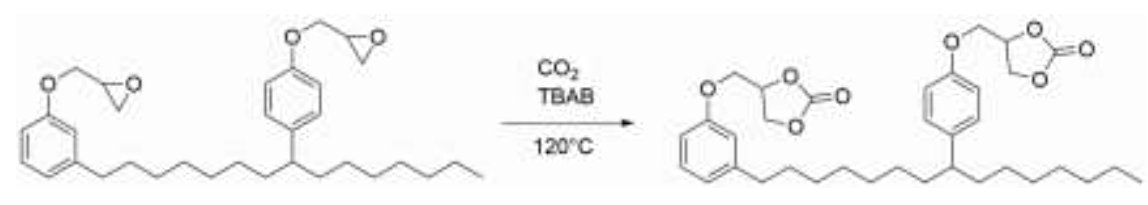

Figure 20. $\mathrm{CO}_{2}$ carbonation of epoxidized Cardanol

Various chemical pathways to synthesize diamines from CNSL have been reported by Wadgaonkar et al. ${ }^{[132]}$ They all proceed through a fastidious multistep protocol. However, a Phenalkamine NC540 (from Cardolite) is commercialized as an epoxy curing agent. ${ }^{[13]}$ This chemical has been recently positively tested for PHU synthesis. ${ }^{\left[{ }^{[3 b]}\right.}$ 
Terpenes are organic compounds produced by plants and trees, particularly from conifers. Limonene, a six-membered ring terpene, has gained interest for the synthesis of renewable building blocks. ${ }^{[133]}$ It is an inexpensive and abundant molecule, which can be readily recovered from orange peel waste. Limonene presents an ideal chemical structure for the synthesis of difunctional monomers (e.g. diamines and CC) with carbon double bonds (Figure 21).

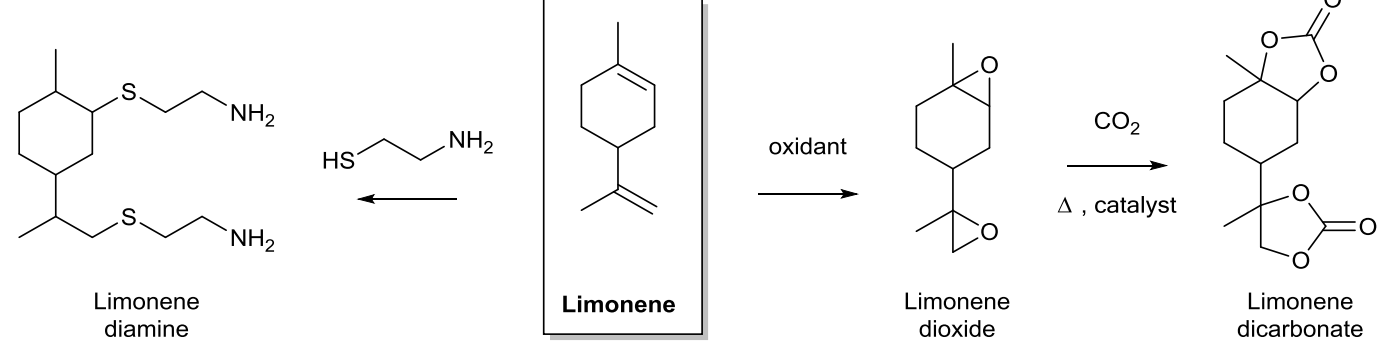

Figure 21. Limonene-based CC and diamines.

Bahr et al. reported the elaboration of CC from a commercially available limonene-based bisepoxide and $\mathrm{CO}_{2}$ at $140^{\circ} \mathrm{C}$ under 30 bars with $\mathrm{TBAB}$ or silica supported iodide catalyst. ${ }^{[134]}$ Even if the heterogeneous catalysts present an easier recovery, they are less efficient with lower activity. However, high purity limonene carbonate could be obtained by crystallization purification. ${ }^{[135]}$ Limonene carbonate has been used with various diamines to obtain linear prepolymers with different molar masses. Their end groups depend on the molar ratio amine/carbonate. The cure of limonene carbonate with polyfunctional amines leads to crosslinked material with high Young's modulus and $\mathrm{T}_{\mathrm{g}}$. No ester groups are present in such compound, thus, no amidification can occur. This is a major benefit in term of control of the reaction and curing procedure.

Diamines from limonene have been reported in 1960 from sulfuric acid and extremely toxic hydrogen cyanide. ${ }^{[136]}$ Since then, a greener route from thiolene click chemistry was studied. The addition of cystamine hydrochloride to limonene, with dimethylpropylamine as catalyst, seems to be an effective way to obtain diamines for biobased PA and PHU (Figure 21). ${ }^{[137]}$ 


\subsection{Synthesis and properties of biobased PHUs}

No specific chemical reactions are particularly elaborated for the synthesis of biobased PHUs. Biobased monomers, such as $\mathrm{CC}$ and diamines, are used in the same conditions than the fossil-based ones. For equivalent structures, there is no difference in term of kinetics, molar masses or secondary reactions. But the environmental impact (LCA) of the final material is modified. Besides, these "new" molecular architectures from the biomass can bring "new" properties.

The main studies about PHUs focus on the CC synthesis. However, amines have great influence on PHUs with a large range of properties. Different amines are thus used with biobased CC to extend the domains of applications. Although the amine structure is major, emphasis is made on renewable CC, since the used amines are often fossil-based. The different used amines are commercialized and only few papers are dedicated to the synthesis of biobased amines for PHUs synthesis. Only few biobased PHUs materials were developed, and furthermore their properties were not often deeply studied and reported.

Below, the trends regarding the "structure-properties" relationships of PHUs materials from biobased CC are discussed. A general overview resuming these relationships is shown in Table 1.

\section{Relationship between resources and final structures}

Reactivity, processing and potential properties of PHUs are directly impacted by the nature of the renewable resources. Thus, parameters such as the chemical structure, the number of reactive sites, the length, the functionality or the state of monomers (liquid vs. solid) drive the structure/properties relationships. The choice of the resource is a key point to target specific properties. For example, vegetable oils are mainly soft with functional compounds, but they possess internal $\mathrm{CC}$ on aliphatic chains which have a low reactivity. ${ }^{[60 \mathrm{~b}]}$ On the other hand, thanks to their aromatic structures, wood derivatives such as lignins or tannins bring stiffness and thermal stability. ${ }^{[120,122]}$ Finally, sugar-based CC are generally short, stiff and reactive monomers. ${ }^{[35,115-116]}$ The nature of the functional sites will also determine syntheses routes. Carbonated vegetable oils are obtained through oxidative epoxidation followed by $\mathrm{CO}_{2}$ carbonation $^{[60]}$ whereas sugar derivatives are usually directly carbonated thanks carbonation 
of vicinal diols. ${ }^{[35,114-116]}$ Finally, CC compounds from wood origin are generally epoxidized via epichlorohydrin addition and $\mathrm{CO}_{2}$ carbonation. ${ }^{[124 \mathrm{a}, 131]}$ The different natures of the obtained CC play a key role on reactivity and possible side-reactions such as amidification. ${ }^{[60 \mathrm{~b}, 70]}$ Thus, molar masses and properties are directly impacted.

\section{Physical properties}

Most of the $\mathrm{CC}$ monomers are bifunctional and very few compounds possess a functionality above 2. Vegetable oil, SOR and some lignin-based monomers can be cited. The access to cross-linked PHUs materials is thus limited so far. The choice of the curing agents is therefore crucial to play on the properties. Mechanical properties are strongly dependent on amine structure, and more particularly on the cross-linking density and hydrogen bonding between urethane, $\mathrm{OH}$, and ester groups of the final macromolecular architectures. PHUs based on rigid cycloaliphatic and aromatic diamines (e.g. IPDA or xylene diamine) display higher tensile stress and lower elongation at break than the materials from linear aliphatic diamines such as ethylene or butane diamines, and HMDA. ${ }^{[60]}$ The same behavior is also encountered when decreasing the carbonated chain length of aliphatic diamines. ${ }^{[60 \mathrm{~b}]}$ Bahr et al. reported the synthesis of PHUs from rigid limonene CC and polyfunctional amines. ${ }^{[134]}$ They showed that increasing the amine functionality enhances Young modulus and limits the elongation at break. Furthermore, when CC and polyfunctional amines are both based on rigid structures, very stiff and brittle thermosetting PHUs are obtained. In the case of PHUs from vegetable oil derived cyclic carbonates, the mechanical properties are strongly dependent on the CC group content. ${ }^{[86]}$ Young modulus and tensile strength increase with raising the carbonate content i.e. with the CC functionality, contrary to the elongation at break. Thus, PHUs from CLSO afford higher strength than those from CSBO. ${ }^{[86]}$

The structures of the diamine and the CC highly influences the $\mathrm{T}_{\mathrm{g}}$ of PHUs. In all papers, PHUs based on linear amines own a lower $\mathrm{T}_{\mathrm{g}}$ compared to cycloaliphatic and aromatic amines ones, which are more rigid. ${ }^{[60,108,134]}$ In addition, the length of the aliphatic carbonated chain also influences the $T_{g}$ value. $T_{g}$ decreases with increasing the carbon number in the diamine aliphatic chain. ${ }^{[86]}$ Limonene-based CC is one of the most rigid monomer encountered in PHU synthesis. Thus, these PHU systems exhibit $\mathrm{T}_{\mathrm{g}}$ higher than $30^{\circ} \mathrm{C}$ whatever the diamine used. ${ }^{[134]}$ Besse et al. also studied the influence of the length of ISO oligomers inside the CC structure, and no noticeable difference in $\mathrm{T}_{\mathrm{g}}$ was revealed. ${ }^{[108]} \mathrm{T}_{\mathrm{g}}$ values of thermosetting PHUs from softer vegetable oils structures are also high due to the polyfunctionality of the 
triglycerides (higher than 2), which leads to a strong cross-linked material. ${ }^{[86]}$ Boyer et al. synthesized PHU from well-defined fatty acid diesters to obtain linear PHU materials with lower $\mathrm{T}_{\mathrm{g}}\left(-15^{\circ} \mathrm{C}\right) .{ }^{[70]}$ These materials are thus more suitable for coatings applications.

Thermal stability of biobased PHUs are similar to conventional PUs with significant degradations above $200^{\circ} \mathrm{C}$, generally at around $250^{\circ} \mathrm{C} .{ }^{[108,}$ 131] Similar behaviors were observed when analyses are performed under inert gas or air, indicating that no specific oxidation phenomenon occurs. ${ }^{[108]}$

\begin{tabular}{|c|c|c|c|c|}
\hline \multirow{2}{*}{ RESOURCES } & \multirow{2}{*}{$\begin{array}{l}\text { EXAMPLES OF STRUCTURES } \\
\text { from renewable starting reagents }\end{array}$} & \multirow{2}{*}{$\begin{array}{l}\text { MAIN SYNTHETIC } \\
\text { ROUTES }\end{array}$} & \multicolumn{2}{|c|}{ PROPERTIES TENDANCIES } \\
\hline & & & $\oiint$ & $\theta$ \\
\hline Vegetableoil & Triglycerides & $\begin{array}{l}>\mathrm{H}_{2} \mathrm{O}_{2} / \mathrm{CO}_{2} \\
>\text { 算 }\end{array}$ & $\begin{array}{l}>\text { High Functionnality } \\
>\text { Liquid state } \\
>\text { Soft materials }\end{array}$ & $\begin{array}{l}>\text { Low reactivity } \\
>\text { Amidifcation }\end{array}$ \\
\hline 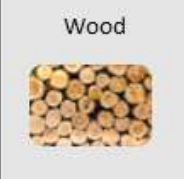 & Cardano & $\begin{array}{l}>\mathrm{H}_{2} \mathrm{O}_{2} / \mathrm{CO}_{2} \\
>\stackrel{\mathrm{i} / \mathrm{CO}_{2}}{>}\end{array}$ & $\begin{array}{l}>\text { Stiffness } \\
>\text { Good thermal stability }\end{array}$ & $>$ Solid state \\
\hline Starch and sugar & Isosorbide & 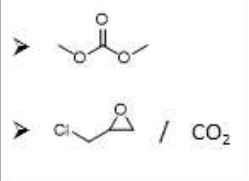 & $\begin{array}{ll}> & \text { Stiffness } \\
> & \text { High reactivity }\end{array}$ & $>$ Solid state \\
\hline
\end{tabular}

Table 1. General illustration of the main relationships between resources and properties for materials based on renewable $\mathrm{CC}$

\section{Conclusions and future trends}

NIPUs are a new promising generation of PUs and have shown a strong and growing interest during the last years. Different routes have been detected but aminolysis reaction has been at the origin of most of NIPU studies.

Nowadays, in agreement with different eco-friendly trends and the research of new macromolecular architectures, PHUs are more and more often biobased. This new class of NIPUs has a huge potential in various environmental applications e.g. building or automotive materials, and adhesives. The interest for biobased PHUs, a very recent topic, is rapidly growing. But until now, the number of publications and patents is still limited. Only few 
potential biobased molecules have been investigated for the development of new sustainable PHUs. The main developments were concentrated on vegetable oils and their derivatives based on a rich oleo-chemistry. Now, new biobased resources receive greater attention. Indeed, contrary to linear structures of triglycerides and fatty acids, building blocks from starch, sugar or lignocellulosic biomass present aromatic or cycloaliphatic structures, that can improve the properties of PHU materials. Furthermore, most of these molecules do not compete with food resources. Unfortunately, only limited data have been published on thermal, structural, and mechanical properties of most of the corresponding polymers.

This review clearly shows that one of the current limitations of biobased PHUs development, as conventional PHUs, still remains the low reactivity of the reaction and the lack of efficient catalysts dedicated to this new PU chemistry. Even if the chemical structures are not exactly equivalent, PHUs can be compared to conventional PUs. PHUs (biobased or not) exhibit in general lower molar masses, slower kinetics, and thus some limited properties for a large range of applications except where low molar mass is required such as adhesives, for instance.

This field of investigations opens a large window for a safer, greener environment, and a healthier future. In summary, all these current developments highlight the high potential of biobased building blocks to develop innovative and performing PHU materials in agreement with the emergent concept of sustainable development. Further improvements could be to adjust the PHU properties with various biobased building blocks in order to fulfill the requirements for several durable applications. Answers could also emerge from investigations around post reactions with the $\mathrm{OH}$ moieties (created by the aminolysis reaction) and mixed systems such as PHU-epoxy for instance.

\section{AKCNOWLEDGEMENTS}

The authors thank Kim Tremblay-Parrado (ICPEES, Strasbourg, France) for the English correction and the Alsace Region and the urban community of Strasbourg (Eurométropole) for the financial support. 


\section{References}

[1] R. Mülhaupt, Macromol. Chem. Phys. 2013, 214, 159-174.

[2] W. Baltus, D. Carrez, M. Carus, H. Kaeb, J. Ravenstijn, S. Zepnik, Nova Institute, Hürth, 2013.

[3] ASTM-International, in Standard Test Methods for Determining the Biobased Content of Solid, Liquid, and Gaseous Samples Using Radiocarbon Analysis, West Conshohocken, PA, 2012.

[4] A. Cornille, R. Auvergne, O. Figovsky, B. Boutevin, S. Caillol, Eur. Polym. J. 2017, $87,535-552$.

[5] a) M. Reulier, R. M. Boumbimba, Z. W. Korb, R. Vaudemont, L. Averous, J. Appl. Polym. Sci. 2017, 134, 44610; b) M. Reulier, R. Perrin, L. Averous, J. Appl. Polym. Sci. 2016, $133,43878$.

[6] a) D. Randall, S. Lee, The polyurethanes book, Distributed by J. Wiley, 2002; b) M. Bock, Polyurethanes for Coatings, Curt R. Vincentz Verlag, 2001.

[7] a) C. Bueno-Ferrer, E. Hablot, M. Del Carmen Garrigos, S. Bocchini, L. Averous, A. Jiménez, Polym. Degrad. Stab. 2012, 97, 1964-1969; b) C. Bueno-Ferrer, E. Hablot, F. Perrin-Sarazin, M. C. Garrigós, A. Jiménez, L. Averous, Macromol. Mater. Eng. 2012, 297, 777-784; c) G. Lligadas, J. C. Ronda, M. Galià, V. Cádiz, Biomacromolecules 2010, 11, 2825-2835; d) D. P. Pfister, Y. Xia, R. C. Larock, ChemSusChem 2011, 4, 703-717; e) O. Kreye, H. Mutlu, M. A. R. Meier, Green Chem. 2013, 15, 1431-1455; f) A. S. More, L. Maisonneuve, T. Lebarbé, B. Gadenne, C. Alfos, H. Cramail, Eur. J.Lipid Sci. Technol. 2013, 115, 61-75.

[8] O. Bayer, Angew Chem-Ger Edit 1947, 59, 257-272.

[9] a) W.-D. Deckwer, FEMS Microbiol. Rev. 1995, 16, 143-149; b) Anonymous, IP.com J. 2013, 13, 1-5; c) O. S. Fruchey, L. E. Manzer, D. Dunuwila, B. T. Keen, B. A. Albin, N. A. Clinton, B. D. Dombek, BioAmber S.A.S., Can. . WO, 2012, p. 30pp.

[10] a) D. Babb, in Synthetic Biodegradable Polymers, Vol. 245 (Eds.: B. Rieger, A. Künkel, G. W. Coates, R. Reichardt, E. Dinjus, T. A. Zevaco), Springer Berlin Heidelberg, 2012, pp. 315-360; b) M. Desroches, M. Escouvois, R. Auvergne, S. Caillol, B. Boutevin, Polym. Rev. 2012, 52, 38-79; c) U. Biermann, U. Bornscheuer, M. A. R. Meier, J. O. Metzger, H. J. Schaefer, Angew. Chem., Int. Ed. 2011, 50, 3854-3871.

[11] a) L. Hojabri, X. Kong, S. S. Narine, Biomacromolecules 2009, 10, 884-891; b) A. S. More, T. Lebarbé, L. Maisonneuve, B. Gadenne, C. Alfos, H. Cramail, Eur. Polym. J. 2013, 49, 823-833; c) G. Çaylı, S. Küsefoğlu, J. Appl. Polym. Sci. 2008, 109, 2948-2955; d) L. Hojabri, X. Kong, S. S. Narine, J. Polym. Sci., Part A: Polym. Chem. 2010, 48, 33023310 .

[12] M. D. Zenner, Y. Xia, J. S. Chen, M. R. Kessler, ChemSusChem 2013, 6, 1182-1185. 
[13] M. Charlon, B. Heinrich, Y. Matter, E. Couzigné, B. Donnio, L. Avérous, Eur. Polym. J. 2014, 61, 197-205.

[14] a) NIOSH, Vol. Publication No. 2004-116, Centers for Disease Control and Prevention, Cincinnati, 2004; b) in Official Journal of the European Union, Vol. L 164, 2009, pp. 7-31; c) X. Baur, W. Marek, J. Ammon, A. B. Czuppon, B. Marczynski, M. Raulf-Heimsoth, H. Roemmelt, G. Fruhmann, Int. Arch. Occup. Environ. Health 1994, 66, 141-152; d) S. M. Arnold, M. A. Collins, C. Graham, A. T. Jolly, R. J. Parod, A. Poole, T. Schupp, R. N. Shiotsuka, M. R. Woolhiser, Regul. Toxicol. Pharmacol; e) J. M. Peters, Proc. R. Soc. Med. 1970, 63, 372-375.

[15] J. Guan, Y. H. Song, Y. Lin, X. Z. Yin, M. Zuo, Y. H. Zhao, X. L. Tao, Q. Zheng, Ind. Eng. Chem. Res. 2011, 50, 6517-6527.

[16] B. Nohra, L. Candy, J.-F. Blanco, C. Guerin, Y. Raoul, Z. Mouloungui, Macromolecules (Washington, DC, U. S.) 2013, 46, 3771-3792.

[17] L. Maisonneuve, O. Lamarzelle, E. Rix, E. Grau, H. Cramail, Chem Rev 2015, 115, 12407-12439.

[18] M. S. Kathalewar, P. B. Joshi, A. S. Sabnis, V. C. Malshe, RSC Adv. 2013, 3, 41104129.

[19] a) D. V. Palaskar, A. Boyer, E. Cloutet, C. Alfos, H. Cramail, Biomacromolecules 2010, 11, 1202-1211; b) E. F. V. Scriven, K. Turnbull, Chem. Rev. 1988, 88, 297-368.

[20] a) P. Tundo, L. Rossi, A. Loris, J. Org. Chem. 2005, 70, 2219-2224; b) P. Deepa, M. Jayakannan, J Polym Sci Pol Chem 2008, 46, 2445-2458; c) M. Jayakannan, D. Puthanparambil, Council of Scientific and Industrial Research, India . US 2007/0117950, 2007, p. 10 pp.

[21] A. S. More, B. Gadenne, C. Alfos, H. Cramail, Polym. Chem. 2012, 3, 1594-1605.

[22] J. H. Clements, Ind. Eng. Chem. Res. 2003, 42, 663-674.

[23] a) J. Nemirowsky, J. Prakt. Chem. 1883, 28, 439-440; b) R. M. Burk, M. B. Roof, Tetrahedron Lett. 1993, 34, 395-398.

[24] R. Darling, P. Rega, Phosgene Toxicity, can be found under http://emedicine.medscape.com/article/832454-overview, 2014.

[25] S. Benyahya, M. Desroches, R. Auvergne, S. Carlotti, S. Caillol, B. Boutevin, Polym. Chem. 2011, 2, 2661-2667.

[26] M. Aresta, A. Dibenedetto, Dalton Trans. 2007, 28, 2975-2992.

[27] a) T. Sakakura, K. Kohno, Chem. Commun. 2009, 11, 1312-1330; b) A. Dibenedetto, A. Angelini, P. Stufano, J. Chem. Technol. Biotechnol. 2014, 89, 334-353.

[28] a) W. Tam, J. Org. Chem. 1986, 51, 2977-2981; b) D. M. Pearson, N. R. Conley, R. M. Waymouth, Adv. Synth. Catal. 2011, 353, 3007-3013. 
[29] S. P. Chavan, B. M. Bhanage, Tetrahedron Lett. 2014, 55, 1199-1202.

[30] M. Doya, K. Kimizuka, Y. Kanbara, Mitsubishi Gas Chemical Company, Inc., US, 1996.

[31] a) B. M. Bhanage, S.-i. Fujita, Y. Ikushima, M. Arai, Green Chem. 2003, 5, 429-432; b) Q. Li, W. Zhang, N. Zhao, W. Wei, Y. Sun, Catal. Today 2006, 115, 111-116.

[32] J. R. Ochoa-Gómez, O. Gómez-Jiménez-Aberasturi, B. Maestro-Madurga, A. Pesquera-Rodríguez, C. Ramírez-López, L. Lorenzo-Ibarreta, J. Torrecilla-Soria, M. C. Villarán-Velasco, Applied Catalysis A: General 2009, 366, 315-324.

[33] S. H. Pyo, R. Hatti-Kaul, Adv. Synth. Catal. 2016, 358, 834-839.

[34] S. Guidi, R. Calmanti, M. Noè, A. Perosa, M. Selva, ACS Sustainable Chemistry \& Engineering 2016, 4, 6144-6151.

[35] S. Schmidt, F. J. Gatti, M. Luitz, B. S. Ritter, B. Bruchmann, R. Mülhaupt, Macromolecules 2017, 50, 2296-2303.

[36] M. North, R. Pasquale, C. Young, Green Chem. 2010, 12, 1514-1539.

[37] K. Vierling, DE 740,366, 1943.

[38] a) Q. He, J. W. O'Brien, K. A. Kitselman, L. E. Tompkins, G. C. T. Curtis, F. M. Kerton, Catal. Sci. Technol. 2014, 4, 1513-1528; b) J. Sun, S.-i. Fujita, M. Arai, J. Organomet. Chem. 2005, 690, 3490-3497; c) W. Cheng, Q. Su, J. Wang, J. Sun, F. T. T. $\mathrm{Ng}$, Catalysts 2013, 3, 878-901, 824 pp.

[39] P. C. Mazo, L. A. Rios, Chem. Eng. J. 2012, 210, 333-338.

[40] J. A. Verdol, Sinclair Refining Co. . US, 1962, p. 2 pp.

[41] a) N. Eghbali, C.-J. Li, Green Chem. 2007, 9, 213-215; b) D. Bai, H. Jing, Green Chem. 2010, 12, 39-41.

[42] a) J. Sun, S.-i. Fujita, B. M. Bhanage, M. Arai, Catal. Today 2004, 93-95, 383-388; b) J. Sun, S.-i. Fujita, F. Zhao, M. Hasegawa, M. Arai, J. Catal. 2005, 230, 398-405.

[43] X. Gao, G. Yuan, H. Chen, H. Jiang, Y. Li, C. Qi, Electrochem. Commun. 2013, 34, $242-245$.

[44] a) K. Tomishige, H. Yasuda, Y. Yoshida, M. Nurunnabi, B. Li, K. Kunimori, Green Chem. 2004, 6, 206-214; b) Y. Du, L.-N. He, D.-L. Kong, Catalysis Communications 2008, 9, 1754-1758; c) Y. Du, D.-L. Kong, H.-Y. Wang, F. Cai, J.-S. Tian, J.-Q. Wang, L.N. He, J. Mol. Catal. A: Chem. 2005, 241, 233-237; d) S. Huang, J. Ma, J. Li, N. Zhao, W. Wei, Y. Sun, Catalysis Communications 2008, 9, 276-280; e) J. George, Y. Patel, S. M. Pillai, P. Munshi, J. Mol. Catal. A: Chem. 2009, 304, 1-7; f) M. Tamura, M. Honda, Y. Nakagawa, K. Tomishige, J. Chem. Technol. Biotechnol. 2014, 89, 19-33.

[45] A. Baba, H. Kashiwagi, H. Matsuda, Tetrahedron Lett. 1985, 26, 1323-1324. 
[46] J. Mindemark, PhD Thesis thesis, Uppsala University 2012.

[47] H. Tomita, F. Sanda, T. Endo, J. Polym. Sci., Part A: Polym. Chem. 2001, 39, 40914100 .

[48] A. Yuen, A. Bossion, E. Gomez-Bengoa, F. Ruiperez, M. Isik, J. L. Hedrick, D. Mecerreyes, Y. Y. Yang, H. Sardon, Polym. Chem. 2016, 7, 2105-2111.

[49] R. Wu, T. F. Al-Azemi, K. S. Bisht, Biomacromolecules 2008, 9, 2921-2928.

[50] A.-A. G. Shaikh, S. Sivaram, Chem. Rev. (Washington, D. C.) 1996, 96, 951-976.

[51] A. Bornadel, R. Hatti-Kaul, K. Sörensen, S. Lundmark, S.-H. Pyo, Biotechnol. Prog. 2013, 29, 66-73.

[52] a) S. Benyahya, B. Boutevin, S. Caillol, V. Lapinte, J.-P. Habas, Polym. Int. 2012, 61, 918-925; b) A. Lachowicz, G. F. Grahe, Dainippon Ink Chemical Industry Co., Japan . DE 3,937,113, 1991, p. 9 pp; c) M. Helou, J.-F. Carpentier, S. M. Guillaume, Green Chem. 2011, 13, 266-271; d) C. Carre, L. Bonnet, L. Averous, RSC Adv. 2014, 4, 54018-54025.

[53] L. Annunziata, A. K. Diallo, S. Fouquay, G. Michaud, F. Simon, J. M. Brusson, J. F. Carpentier, S. M. Guillaume, Green Chem. 2014, 16, 1947-1956.

[54] C. N. Tang, H. B. Nulwala, K. Damodaran, P. Kaur, D. R. Luebke, Journal of Polymer Science, Part A: Polymer Chemistry 2011, 49, 2024-2032.

[55] R. M. Garipov, V. A. Sysoev, V. V. Mikheev, A. I. Zagidullin, R. Y. Deberdeev, V. I. Irzhak, A. A. Berlin, Dokl. Phys. Chem. 2003, 393, 289-292.

[56] C. D. Diakoumakos, D. L. Kotzev, Macromol. Symp. 2004, 216, 37-46.

[57] F. Camara, S. Benyahya, V. Besse, G. Boutevin, R. Auvergne, B. Boutevin, S. Caillol, Eur. Polym. J. 2014, 55, 17-26.

[58] H. Tomita, F. Sanda, T. Endo, Journal of Polymer Science, Part A: Polymer Chemistry 2001, 39, 3678-3685.

[59] A. Cornille, M. Blain, R. Auvergne, B. Andrioletti, B. Boutevin, S. Caillol, Polym. Chem. 2017, 8, 592-604.

[60] a) I. Javni, D. P. Hong, Z. S. Petrović, J. Appl. Polym. Sci. 2013, 128, 566-571; b) I. Javni, D. P. Hong, Z. S. Petrovic, J. Appl. Polym. Sci. 2008, 108, 3867-3875.

[61] a) E. Delebecq, J.-P. Pascault, B. Boutevin, F. Ganachaud, Chem. Rev. (Washington, $D C, U$. S.) 2013, 113, 80-118; b) O. Figovsky, L. Shapovalov, A. Leykin, O. Birukova, R. Potashnikova, Int. Lett. Chem., Phys. Astron. 2012, 3, 52-66.

[62] R. H. Lambeth, T. J. Henderson, Polymer 2013, 54, 5568-5573.

[63] D. L. Tang, D. J. Mulder, B. A. J. Noordover, C. E. Koning, Macromol. Rapid Commun. 2011, 32, 1379-1385. 
[64] B. Ochiai, S. Inoue, T. Endo, Journal of Polymer Science, Part A: Polymer Chemistry 2005, 43, 6282-6286.

[65] M. Blain, L. Jean-Gérard, R. Auvergne, D. Benazet, S. Caillol, B. Andrioletti, Green Chem. 2014, 16, 4286-4291.

[66] T. Bürgel, M. Fedtke, Polym. Bull. 1991, 27, 171-177.

[67] V. Besse, F. Camara, F. Méchin, E. Fleury, S. Caillol, J.-P. Pascault, B. Boutevin, Eur. Polym. J. 2015, 71, 1-11.

[68] B. Nohra, L. Candy, J.-F. Blanco, Y. Raoul, Z. Mouloungui, J. Am. Oil Chem. Soc. 2012, 89, 1125-1133.

[69] B. Tamami, S. Sohn, G. L. Wilkes, J. Appl. Polym. Sci. 2004, 92, 883-891.

[70] A. Boyer, E. Cloutet, T. Tassaing, B. Gadenne, C. Alfos, H. Cramail, Green Chem. 2010, 12, 2205-2213.

[71] M. Blain, A. Cornille, B. Boutevin, E. Auvergne, D. Benazet, B. Andrioletti, S. Caillol, J. Appl. Polym. Sci. 2017, 134, 44958.

[72] H. Tomita, F. Sanda, T. Endo, Journal of Polymer Science, Part A: Polymer Chemistry 2001, 39, 851-859.

[73] F. Cherubini, Energy Convers. Manage. 2010, 51, 1412-1421.

[74] M. Pelckmans, T. Renders, S. Van de Vyver, B. F. Sels, Green Chem. 2017, 19, 5303 5331.

[75] F. D. Gunstone, J. L. Harwood, F. B. Padley, Eur. J. Lipid Sci. Technol. 1995, 97, 315-316.

[76] N. Mann, S. K. Mendon, J. W. Rawlins, S. F. Thames, J. Am. Oil Chem. Soc. 2008, $85,791-796$.

[77] M. F. Lie Ken Jie, C. Yan-Kit, Lipids 1988, 23, 367-369.

[78] a) M. Rüsch gen. Klaas, S. Warwel, Ind. Crops Prod. 1999, 9, 125-132; b) F. Bjorkling, S. E. Godtfredsen, O. Kirk, J. Chem. Soc., Chem. Commun. 1990, 1301-1303.

[79] L. Zhang, Y. Luo, Z. Hou, Z. He, W. Eli, J. Am. Oil Chem. Soc. 2014, 91, 143-150.

[80] X. Wu, X. Zhang, S. Yang, H. Chen, D. Wang, J. Am. Oil Chem. Soc. 2000, 77, 561563.

[81] K. Carlson, R. Kleiman, M. Bagby, J Am Oil Chem Soc 1994, 71, 175-182.

[82] D. Miloslavskiy, E. Gotlib, O. Figovsky, D. D. Pashin, Int. Lett. Chem., Phys. Astron. 2014, 8, 20-29. 
[83] a) A. R. Mahendran, G. Wuzella, N. Aust, U. Müller, J Coat Technol Res 2014, 11, 329-339; b) A. R. Mahendran, N. Aust, G. Wuzella, U. Mueller, A. Kandelbauer, J. Polym. Environ. 2012, 20, 926-931.

[84] Z. Li, Y. Zhao, S. Yan, X. Wang, M. Kang, J. Wang, H. Xiang, Catal. Lett. 2008, 123, 246-251.

[85] P. G. Parzuchowski, M. Jurczyk-Kowalska, J. Ryszkowska, G. Rokicki, J. Appl. Polym. Sci. 2006, 102, 2904-2914.

[86] M. Bahr, R. Mulhaupt, Green Chem. 2012, 14, 483-489.

[87] P. Mazo, L. Rios, J. Am. Oil Chem. Soc. 2013, 90, 725-730.

[88] K. M. Doll, S. Z. Erhan, Green Chem. 2005, 7, 849-854.

[89] S. Samanta, S. Selvakumar, J. Bahr, D. S. Wickramaratne, M. Sibi, B. J. Chisholm, ACS Sustainable Chemistry \& Engineering 2016, 4, 6551-6561.

[90] A. Z. Yu, R. A. Setien, J. M. Sahouani, J. Docken, D. C. Webster, J. Coat. Technol. Res. 2018.

[91] L. Maisonneuve, A. S. More, S. Foltran, C. Alfos, F. Robert, Y. Landais, T. Tassaing, E. Grau, H. Cramail, RSC Adv. 2014, 4, 25795-25803.

[92] M. Stemmelen, F. Pessel, V. Lapinte, S. Caillol, J. P. Habas, J. J. Robin, J. Polym. Sci., Part A: Polym. Chem. 2011, 49, 2434-2444.

[93] a) M. Capelot, $\mathrm{PhD}$ thesis thesis, Université Pierre et Marie Curie (Paris), 2013; b) A. Aboudzadeh, D. Mecerreyes, in EUPOC2013 on Polymers \& Ionic Liquids, Gargnano, 2013; c) A. Aboudzadeh, M. Fernandez, M. E. Munoz, A. Santamaria, D. Mecerreyes, Macromol. Rapid Commun. 2014, 35, 460-465.

[94] T. Werpy, G. Petersen,Top Value Added Chemicals from Biomass Vol. 1, 2004.

[95] A. Behr, J. Eilting, K. Irawadi, J. Leschinski, F. Lindner, Green Chem. 2008, 10, 13 30.

[96] M. O. Sonnati, S. Amigoni, E. P. Taffin de Givenchy, T. Darmanin, O. Choulet, F. Guittard, Green Chem. 2013, 15, 283-306.

[97] J. R. Ochoa-Gomez, O. Gomez-Jimenez-Aberasturi, C. Ramirez-Lopez, M. Belsue, Org. Process Res. Dev. 2012, 16, 389-399.

[98] K. Lanjekar, V. K. Rathod, J. Environ. Chem. Eng. 2013, 1, 1231-1236.

[99] A. R. Go, Y. Lee, Y. H. Kim, S. Park, J. Lee, S. O. Han, S. W. Kim, C. Park, Enzyme Microb. Technol. 2013, 53, 154-158.

[100] M. Tryznowski, A. Świderska, Z. Żołek-Tryznowska, T. Gołofit, P. G. Parzuchowski, Polymer 2015, 80, 228-236. 
[101] Y. J. Li, J. X. Liu, D. H. He, Appl Catal a-Gen 2018, 564, 234-242.

[102] a) J. L. J. van Velthoven, L. Gootjes, D. S. van Es, B. A. J. Noordover, J. Meuldijk, Eur. Polym. J. 2015, 70, 125-135; b) M. Tryznowski, A. Swiderska, T. Golofit, Z. ZolekTryznowska, $R S C A d v$. 2017, 7, 30385-30391.

[103] F. Guibe, Y. S. M'Leux, Tetrahedron Lett. 1981, 22, 3591-3594.

[104] N. Bassam, C. Laure, B. Jean-Francois, R. Yann, M. Zephirin, Green Chem. 2013, $15,1900-1909$.

[105] M. Rose, R. Palkovits, ChemSusChem 2012, 5, 167-176.

[106] F. Fenouillot, A. Rousseau, G. Colomines, R. Saint-Loup, J. P. Pascault, Prog. Polym. Sci. 2010, 35, 578-622.

[107] R. Auvergne, S. Caillol, G. David, B. Boutevin, J.-P. Pascault, Chem. Rev. 2013, $114,1082-1115$.

[108] V. Besse, R. Auvergne, S. Carlotti, G. Boutevin, B. Otazaghine, S. Caillol, J.-P. Pascault, B. Boutevin, React. Funct. Polym. 2013, 73, 588-594.

[109] R. Montgomery, L. F. Wiggins, J. Chem. Soc. 1946, 1946, 393-396.

[110] J. Thiem, H. Lüders, Makromol. Chem. 1986, 187, 2775-2785.

[111] S. Thiyagarajan, L. Gootjes, W. Vogelzang, J. van Haveren, M. Lutz, D. S. van Es, ChemSusChem 2011, 4, 1823-1829.

[112] a) A. A. Caouthar, A. Loupy, M. Bortolussi, J.-c. Blais, L. Dubreucq, A. Meddour, J. Polym. Sci., Part A: Polym. Chem. 2005, 43, 2480-2491; b) A. Caouthar, P. Roger, M. Tessier, S. Chatti, J. C. Blais, M. Bortolussi, Eur. Polym. J. 2007, 43, 220-230.

[113] J. P. Gillet, WO 145921 A2, 2008.

[114] S. Schmidt, B. S. Ritter, D. Kratzert, B. Bruchmann, R. Mülhaupt, Macromolecules 2016, 49, 7268-7276.

[115] S. Schmidt, N. E. Goppert, B. Bruchmann, R. Mulhaupt, Eur. Polym. J. 2017, 94, $136-142$

[116] P. Furtwengler, L. Averous, Sci Rep 2018, 8, 9134.

[117] L. Zhang, X. L. Luo, Y. S. Qin, Y. B. Li, RSC Adv. 2017, 7, 37-46.

[118] A. Lee, Y. Deng, Eur. Polym. J. 2015, 63, 67-73.

[119] A. Salanti, L. Zoia, M. Orlandi, Green Chem. 2016, 18, 4063-4072.

[120] A. Salanti, L. Zoia, M. Mauri, M. Orlandi, RSC Adv. 2017, 7, 25054-25065.

[121] I. Kühnel, B. Saake, R. Lehnen, Macromol. Chem. Phys. 2018, 219, 1700613. 
[122] a) M. Thébault, A. Pizzi, S. Dumarçay, P. Gerardin, E. Fredon, L. Delmotte, Ind. Crops Prod. 2014, 59, 329-336; b) M. Thébault, A. Pizzi, H. A. Essawy, A. Barhoum, G. Van Assche, Eur. Polym. J. 2015, 67, 513-526.

[123] G. Liu, G. Wu, J. Chen, S. Huo, C. Jin, Z. Kong, Polym. Degrad. Stab. 2015, 121, 247-252.

[124] a) M. Fache, E. Darroman, V. Besse, R. Auvergne, S. Caillol, B. Boutevin, Green Chem. 2014, 16, 1987-1998; b) E. A. B. d. Silva, M. Zabkova, J. D. Araújo, C. A. Cateto, M. F. Barreiro, M. N. Belgacem, A. E. Rodrigues, Chem. Eng. Res. Des. 2009, 87, 12761292.

[125] C. Aouf, J. Lecomte, P. Villeneuve, E. Dubreucq, H. Fulcrand, Green Chem. 2012, $14,2328-2336$.

[126] D. Balgude, A. S. Sabnis, J. Coat. Technol. Res. 2014, 11, 169-183.

[127] V. S. Balachandran, S. R. Jadhav, P. K. Vemula, G. John, Chem. Soc. Rev. 2013, 42, 427-438.

[128] D. J. Garkal, R. S. Bhande, IJIERT 2014, 1, 1-8.

[129] C. Voirin, S. Caillol, N. V. Sadavarte, B. V. Tawade, B. Boutevin, P. P. Wadgaonkar, Polym. Chem. 2014, 5, 3142-3162.

[130] K. I. Suresh, V. S. Kishanprasad, Ind. Eng. Chem. Res. 2005, 44, 4504-4512.

[131] M. Kathalewar, A. Sabnis, D. D’Mello, Eur. Polym. J. 2014, 57, 99-108.

[132] a) A. S. More, P. S. Sane, A. S. Patil, P. P. Wadgaonkar, Polym. Degrad. Stab. 2010, 95, 1727-1735; b) A. S. More, A. S. Patil, P. P. Wadgaonkar, Polym. Degrad. Stab. 2010, 95, 837-844.

[133] P. A. Wilbon, F. Chu, C. Tang, Macromol. Rapid Commun. 2013, 34, 8-37.

[134] M. Bahr, A. Bitto, R. Mulhaupt, Green Chem. 2012, 14, 1447-1454.

[135] V. Schimpf, B. S. Ritter, P. Weis, K. Parison, R. Mulhaupt, Macromolecules 2017, 50, 944-955.

[136] C. H. Mckeever, R. N. Washburne, US 2,955,138A, 1960.

[137] M. Firdaus, M. A. R. Meier, Green Chem. 2013, 15, 370-380. 